\title{
Herpetofauna da área do Igarapé Esperança na Reserva Extrativista Riozinho da Liberdade, Acre - Brasil
}

\author{
Paulo Sérgio Bernarde ${ }^{1,2}$, Reginaldo Assêncio Machado ${ }^{1}$ \& Luiz Carlos Batista Turci ${ }^{1}$ \\ ${ }^{1}$ Laboratório de Herpetologia, Centro Multidisciplinar, Universidade Federal do Acre - UFAC, \\ Campus Floresta, CEP 69980-000, Cruzeiro do Sul, AC, Brasil \\ ${ }^{2}$ Autor para correspondência: Paulo Sérgio Bernarde, e-mail: snakebernarde@hotmail.com
}

BERNARDE, P.S., MACHADO, R.A. \& TURCI, L.C.B. Herpetofauna of Igarapé Esperança area in the Reserva Extrativista Riozinho da Liberdade, Acre - Brazil. Biota Neotrop. 11(3): http://www.biotaneotropica. org.br/v11n3/en/abstract?inventory+bn02111032011

\begin{abstract}
The upper Juruá region is located in Acre state (western Brazilian Amazonia) and it is known for having a high diversity and moreover is considered a priority area for survey and conservation of herpetofauna. This study was carried out from August 2006 to June 2008 and presents a list of amphibians and reptiles from Igarapé Esperança area in the Reserva Extrativista Riozinho da Liberdade, Acre. Four sampling methods were used to survey herpetofauna: time constrained visual search, pitfall traps with drift fences, call records and accidental sightings. One hundred and sixty-two species were recorded, including 83 amphibians (80 frogs, two caecilians and one salamander) and 79 reptiles (29 lizards, 42 snakes, one anfisbaenian, four chelonians and three caimans). Of these species, eleven (Adelphobates quinquevittatus, Hyalinobatrachium munozorum, Pristimantis academicus, P. aureolineatus, Syncope antenori, Alopoglossus buckleyi, Drymobius rhombifer, Liophis dorsocorallinus, L. taeniogaster, Umbrivaga pygmaea, e Micrurus remotus) were registered for the first time to Acre, six (H. munozorum, P. academicus, P. aureolineatus, S. antenori, A. buckleyi e L. dorsocorallinus) of which also are the first records for Brazil. The importance of the form of forest use by traditional peoples (indigenous, extractive and riverine people) was pointed out, as well that of areas for conservation of the high biodiversity found in the Upper Juruá. Also the need for studies on the use of some species (especially chelonians and crocodilians) to evaluate possible impacts on the populations of these animals is stressed.
\end{abstract}

Keywords: Amazon, Amphibia, Reptilia, Anura, Squamata.

BERNARDE, P.S., MACHADO, R.A. \& TURCI, L.C.B. Herpetofauna da área do Igarapé Esperança na Reserva Extrativista Riozinho da Liberdade, Acre - Brasil. Biota Neotrop. 11(3): http://www.biotaneotropica. org.br/v11n3/pt/abstract?article+bn02111032011

Resumo: A região do Alto Juruá localiza-se no estado do Acre (oeste da Amazônia brasileira) e é uma região conhecida por apresentar uma alta diversidade e também considerada prioritária para inventariamento e conservação da herpetofauna. Este trabalho foi realizado entre agosto de 2006 a junho de 2008 e apresenta a lista de espécies de anfíbios e répteis da área do Igarapé Esperança na Reserva Extrativista Riozinho da Liberdade, Acre. Quatro métodos amostrais foram empregados para inventariar a herpetofauna: procura limitada por tempo, armadilhas de interceptação e queda, registros auditivos e encontros ocasionais. Foram registradas 162 espécies, sendo 83 de anfíbios (80 anuros, dois gimnofionos e uma salamandra) e 79 de répteis (29 lagartos, 42 serpentes, um anfisbênio, quatro quelônios e três jacarés). Dessas espécies, onze (Adelphobates quinquevittatus, Hyalinobatrachium munozorum, Pristimantis academicus, P. aureolineatus, Syncope antenori, Alopoglossus buckleyi, Drymobius rhombifer, Liophis dorsocorallinus, L. taeniogaster, Umbrivaga pygmaea e Micrurus remotus) foram registradas pela primeira vez para o Acre, sendo que seis delas (H. munozorum, P. academicus, P. aureolineatus, S. antenori, A. buckleyi e L. dorsocorallinus) consistem também os primeiros registros para o Brasil. Salienta-se aqui a importância da forma do uso das florestas pelas populações tradicionais (indígenas, extrativistas e ribeirinhos) e das áreas protegidas na conservação da alta biodiversidade encontrada no Alto Juruá e também a necessidade de estudos sobre o uso de algumas espécies (especialmente de quelônios e crocodilianos) para analisar possíveis impactos sobre as populações desses animais.

Palavras-chave: Amazônia, Amphibia, Reptilia, Anura, Squamata. 


\section{Introdução}

O Brasil apresenta a maior riqueza de anfíbios do Planeta (SBH 2010) e a segunda de répteis (Bérnils 2010), com respectivamente 875 e 721 espécies. Grande parte dessas espécies ocorre na Amazônia, onde foram registradas aproximadamente 232 de anfíbios e 273 de répteis (Avila-Pires et al. 2007). Essas riquezas provavelmente se encontram subestimadas, uma vez que várias regiões na Amazônia permanecem subamostradas ou mesmo não amostradas (AzevedoRamos \& Gallati 2001, Vogt et al. 2001). Um dos indicadores da carência de estudos herpetofaunísticos nesse bioma é o fato de ser relativamente comum a publicação de descrição de novas espécies (e. g., Peloso \& Sturaro 2008, Simões et al. 2010) e de novos registros de ocorrência para o país (e. g., Toledo et al. 2009, Avila-Pires et al. 2010, Cisneros-Heredia et al. 2010, Sampaio \& Souza 2010).

Na Amazônia brasileira, os estudos sobre composição faunística de anfíbios e répteis foram desenvolvidos em sua maioria na região de Manaus (e. g., Zimmerman \& Rodrigues 1990, Martins 1991, Martins \& Oliveira 1998, Lima et al. 2006, Menin et al. 2007, Vitt et al. 2008), Leste do Pará (e. g., Cunha \& Nascimento 1993, Avila-Pires \& Hoogmoed 1997, Prudente \& Santos-Costa 2005, Maschio et al. 2009) e no estado de Rondônia (e. g., Vanzolini 1986, Nascimento et al. 1988, Jorge-da-Silva Junior 1993, Bernarde \& Abe 2006, Bernarde 2007, Bernarde \& Macedo 2008, Macedo et al. 2008, Avila-Pires et al. 2009). Apesar da concentração dessas pesquisas no Amazonas, Pará e Rondônia, várias localidades nesses estados carecem de inventários herpetofaunísticos (Azevedo-Ramos \& Gallati 2001, Vogt et al. 2001), carência essa também observada para o estado do Acre.

A região do Alto Juruá localiza-se no estado do Acre e é uma das regiões consideradas prioritárias para inventariamentos e conservação da herpetofauna (Azevedo-Ramos \& Gallati 2001, Vogt et al. 2001), devido a sua alta diversidade, poucos estudos desenvolvidos e sua localização no extremo ocidental da Amazônia brasileira. Dentre os estudos desenvolvidos no Alto Juruá destaca-se o trabalho de Souza (2009) que registrou na Serra do Divisor (Parque Nacional da Serra do Divisor e Reserva Extrativista do Alto Juruá) a maior riqueza de anfíbios (126 espécies) conhecida para uma região no Planeta. Apesar dessa riqueza, foram poucos os estudos desenvolvidos sobre os anfíbios e répteis no Alto Juruá. Dentre eles, algumas novas espécies foram descritas de anfíbios (Martins \& Cardoso 1987, Caldwell 2005) e de lagarto (Avila-Pires \& Vitt 1998). Souza \& Haddad (2003) redescreveram Leptodactylus dantasi e incluíram essa espécie no gênero Hydrolaetare baseados em espécimes coletados na Serra do Divisor. Cardoso \& Vielliard (1990) estudaram a atividade de vocalização de anuros em área aberta em Cruzeiro do Sul. Em Porto Walter, Vitt et al. (1998) estudaram o impacto da formação de clareiras devido a retirada de madeira sobre a fauna de lagartos. Nessa mesma localidade a fauna de répteis Squamata foi inventariada por Avila-Pires et al. (2009), onde a composição de lagartos foi comparada com outras três localidades amazônicas. Turci et al. (2009) estudaram a atividade e uso do habitat por duas serpentes Viperidae (Bothriopsis bilineata e Bothrops atrox) na floresta do Rio Môa, em Cruzeiro do Sul. Bernarde et al. (2010) registraram pela segunda vez para o Brasil o anfíbio anuro Hemiphractus helioi.

As reservas extrativistas foram originadas no Acre no final da Década de 80 a partir dos conflitos entre seringueiros que sobreviviam na floresta (através do extrativismo, pequenos roçados, caça e pesca) e fazendeiros de gado que dependiam do desmatamento para suas atividades (Almeida 2004). Em 2000 foi criado o Sistema Nacional de Unidades de Conservação (SNUC), o qual define as reservas extrativistas (Resex) como unidades que tem por objetivo proteger os meios de vida e garantir a utilização e a conservação dos recursos naturais renováveis tradicionalmente utilizados pela população extrativista residente na área de sua abrangência (Rylands \& Brandon
2005). As atividades de uso sustentável das comunidades tradicionais nas reservas extrativistas ajustadas empiricamente à dinâmica da renovação do sistema pode até contribuir para o aumento da diversidade biológica (Brown Junior \& Freitas 2002), além da importância da manutenção da floresta e de seus serviços ambientais (Ferreira et al. 2005, Fearnside 2006). Entretanto, a presença humana e de mamíferos domésticos em unidades de conservação podem ocasionar impactos negativos sobre algumas espécies da fauna devido a alta pressão de caça, coleta e de risco de transmissão de patógenos, além de outros (Peres 2000, Fachín-Terán et al. 2003, Whiteman et al. 2008). A Reserva Extrativista do Riozinho da Liberdade foi criada a partir do Decreto-Lei de 17 de fevereiro de 2005, ocupando uma área de 325.602 ha nos estados do Acre e Amazonas (Acre 2006), região do Alto Juruá conhecida pela sua alta biodiversidade e também da diversidade sociocultural composta por comunidades tradicionais conhecidas como "povos da floresta" que vivem de pequenos roçados, extrativismo, caça e pesca (Almeida 2004, Brown Junior \& Freitas 2002).

O presente estudo tem como objetivo apresentar a lista de espécies de anfíbios e répteis que ocorrem na área do Igarapé Esperança na Reserva Extrativista do Riozinho da Liberdade, apresentando além da riqueza para essa localidade, novos registros para o Acre e para o Brasil.

\section{Material e Métodos}

Área de estudo: A Reserva Extrativista Riozinho da Liberdade está localizada no extremo oeste do Brasil (Figura 1) nos municípios de Cruzeiro do Sul, Marechal Thaumaturgo, Porto Walter e Tarauacá (Acre), e Ipixuna (Amazonas) (Acre 2006). Seu principal rio é o Liberdade, afluente do Juruá (Acre 2006). A vegetação é representada pela Floresta Ombrófila Aberta, sendo caracterizada pelo dossel normalmente aberto e pela abundância de palmeiras, cipós ou bambus (Acre 2006). Ao longo do Rio Liberdade existem moradores, cujas residências estão inseridas em clareiras onde existem pequenos roçados, principalmente de "macaxeira" (Manihot esculenta Crantz) e as tradicionais casas de farinhas. O clima da região é caracterizado como tropical, quente, úmido, com temperatura média anual de $24{ }^{\circ} \mathrm{C}$ (Ribeiro 1977), sendo período entre os meses de maio a outubro o mais seco do ano.

O estudo foi desenvolvido entre agosto de 2006 a junho de 2008 em uma área de aproximadamente 200 ha no Igarapé Esperança $\left(07^{\circ}\right.$ 57' 20" S e 72 04' 37" O), tributário do Rio Liberdade. Durante agosto a dezembro de 2006 foi realizado o reconhecimento da área de estudo, abertura de trilhas em floresta e construção das armadilhas de interceptação e queda. Durante o período de 18 meses (janeiro de 2007 a junho de 2008) foram realizadas viagens mensais com duração de seis dias, onde a herpetofauna foi registrada através de procura limitada por tempo, armadilhas de interceptação e queda, registros auditivos (anuros) e encontros ocasionais.

\section{Esforço amostral}

Procura visual limitada por tempo (ver Campbell \& Christman 1982, Martins \& Oliveira 1998): Foram percorridas trilhas (de 400 a $500 \mathrm{~m}$ ) que passavam por alguns ambientes aquáticos (igapós, igarapés e poças temporárias) durante a noite (entre 18:00 e 23:00 horas) e manhã (7:00 e 11:00 horas) em um total de 720 horas de procura, sendo 540 horas no período noturno e 180 horas no período diurno.

Armadilhas de interceptação e queda (ver Fitch 1987, Cechin \& Martins 2000): Cada armadilha foi constituída por quatro baldes plásticos de $100 \mathrm{~L}$, enterrados a cada dez metros e ligados por uma cerca de lona preta de um metro de altura que passava sobre os mesmos. Dez armadilhas foram instaladas totalizando 40 baldes, 
sendo cinco em disposição radial "Y" e outras cinco em linha reta. As armadilhas permaneceram abertas cinco dias por mês durante os 18 meses, totalizando 90 dias de amostragem. As armadilhas foram monitoradas todos os dias pela manhã.

Registros auditivos (ver Heyer et al. 1994): Durante as atividades de campo, foram registradas as espécies de anfíbios anuros em atividade de vocalização.

Encontros ocasionais (ver Martins \& Oliveira 1998): Foram registrados os espécimes coletados ou observados ocasionalmente durante as atividades de campo.

Os espécimes foram identificados a partir de chaves, descrições e fotografias presentes nas seguintes bibliografias: Duellman (1978), Dixon \& Soini (1986), Jorge-da-Silva Junior (1993), Cunha \& Nascimento (1993), Duellman \& Rodriguez (1994), Avila-Pires (1995), Martins \& Oliveira (1998), De La Riva et al. (2000), Fernandes et al. (2002), Campbell \& Lamar (2004), Passos \& Fernandes (2005), Esqueda et al. (2005), Guayasamin et al. (2006), Lima et al. (2006), Bergmann \& Russel (2007), Cisneros-Heredia \& McDiarmid (2007), Peloso \& Sturaro (2008), Funk et al. (2008), Prudente et al. (2008), Vitt et al. (2008), Lynch (2009), Souza (2009), Lima \& Prudente (2009), Padial \& De La Riva (2009) e CastroviejoFisher et al. (2009).

Espécimes testemunhos (ver relação em Apêndice) foram depositados na Coleção Herpetológica da Universidade Federal do Acre, Campus Floresta, em Cruzeiro do Sul (UFACF).

\section{Resultados}

Foram registradas 162 espécies na área do Igarapé Esperança na Reserva Extrativista Riozinho da Liberdade (Acre), sendo 83 de anfíbios e 79 de répteis (Ver Lista a seguir e fotografias de algumas espécies nas Figuras 2 a 19). As curvas acumuladas de espécies para anuros e lagartos atingiram a assíntota (Figura 20), entretanto para serpentes não. Em Amphibia registrou-se uma espécie de Urodela, duas de Gymnophiona e 80 de Anura (11 famílias). Dentre os anuros, a família com maior número de espécies foi Hylidae com 36 espécies, seguida de Strabomantidae ( $n=11$ espécies), Leptodactylidae $(n=8)$, Bufonidae $(n=6)$ e Dendrobatidae $(n=6)$, Microhylidae $(n=5)$, Aromobatidae $(\mathrm{n}=3)$, Leiuperidae $(\mathrm{n}=2)$ e as demais com uma espécie: Centrolenidae, Ceratophryidae e Hemiphractidae.

Reptilia foi representada por Testudines (três famílias e quatro espécies), Crocodylia (uma família e três espécies) e Squamata (17 famílias e 72 espécies). Os Squamata foram representados por 42 espécies de Serpentes, 29 de Sauria e uma de Amphisbaenia. Dentre as serpentes, a família com maior número de espécies foi Dipsadidae com 22 espécies, seguida de Colubridae $(\mathrm{n}=11)$, Boidae $(\mathrm{n}=3)$ e Elapidae $(\mathrm{n}=3)$, Viperidae $(\mathrm{n}=2)$ e Aniliidae $(\mathrm{n}=1)$. Gymnophthalmidae e Polychrotidae foram as famílias de lagartos com maior número de espécies (ambas com 6 espécies), seguida de Teiidae $(n=5)$, Sphaerodactylidae $(n=3)$ e Tropiduridae $(n=3)$, Hoplocercidae $(\mathrm{n}=2)$ e Iguanidae, Gekkonidae, Phyllodactylidae e Scincidae (todas com uma espécie).

$68^{\circ} \mathrm{O}$

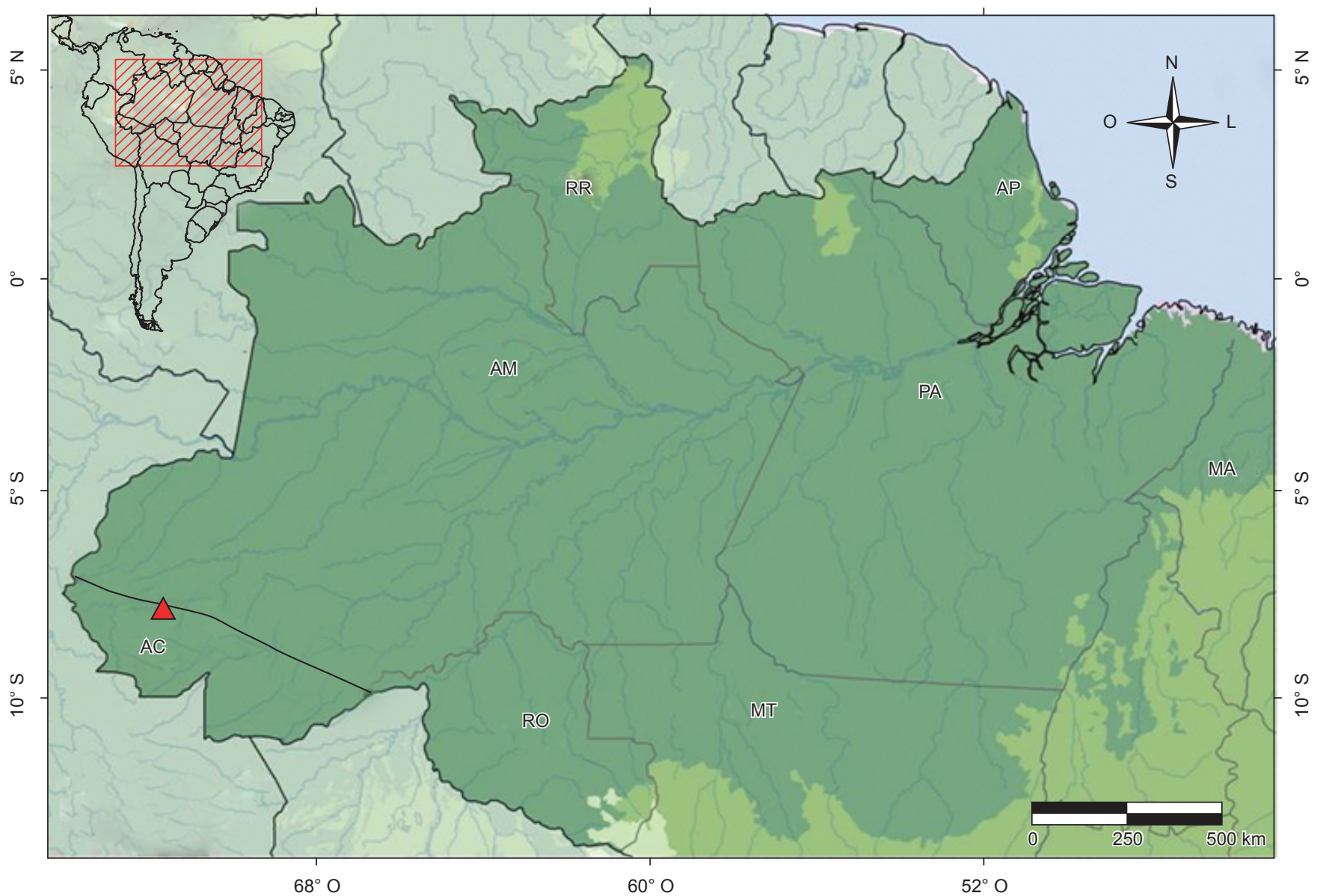

Figura 1. Mapa do Brasil com a localização da Reserva Extrativista Riozinho da Liberdade - Acre.

Figure 1. Map of Brazil with the localization of Reserva Extrativista Riozinho da Liberdade - Acre. 

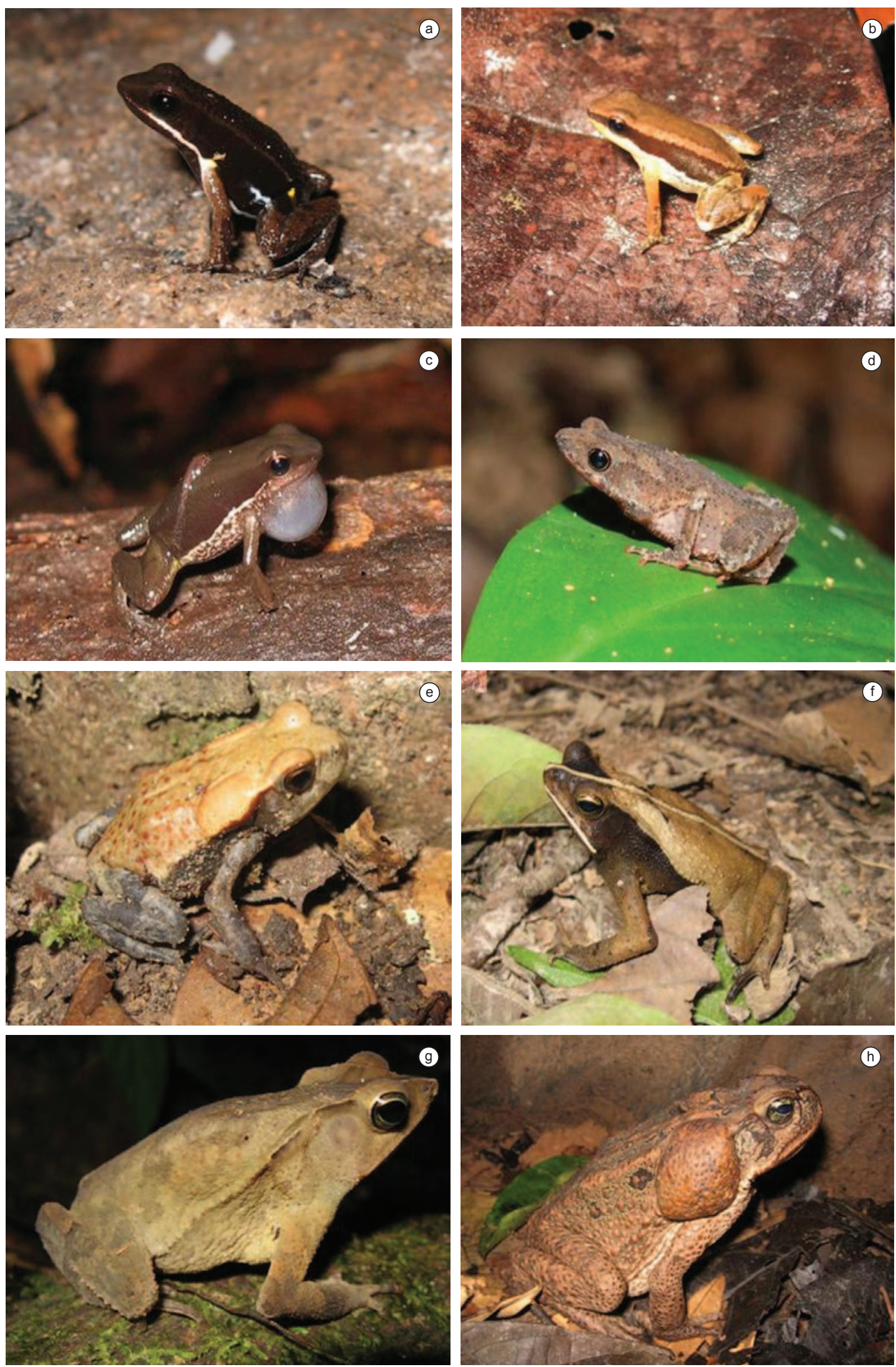

Figura 2. Algumas espécies registradas na Resex Riozinho da Liberdade (AC). a) Allobates femoralis; b) Allobates sp.1; c) Allobates sp.2; d) Dendrophryniscus minutus; e) Rhaebo guttatus; f) Rhinella castaneotica; g) Rhinella margaritifera; h) Rhinella marina.

Figure 2. Some species recorded in the Resex Riozinho da Liberdade (AC). a) Allobates femoralis; b) Allobates sp.1; c) Allobates sp.2; d) Dendrophryniscus minutus; e) Rhaebo guttatus; f) Rhinella castaneotica; g) Rhinella margaritifera; h) Rhinella marina 

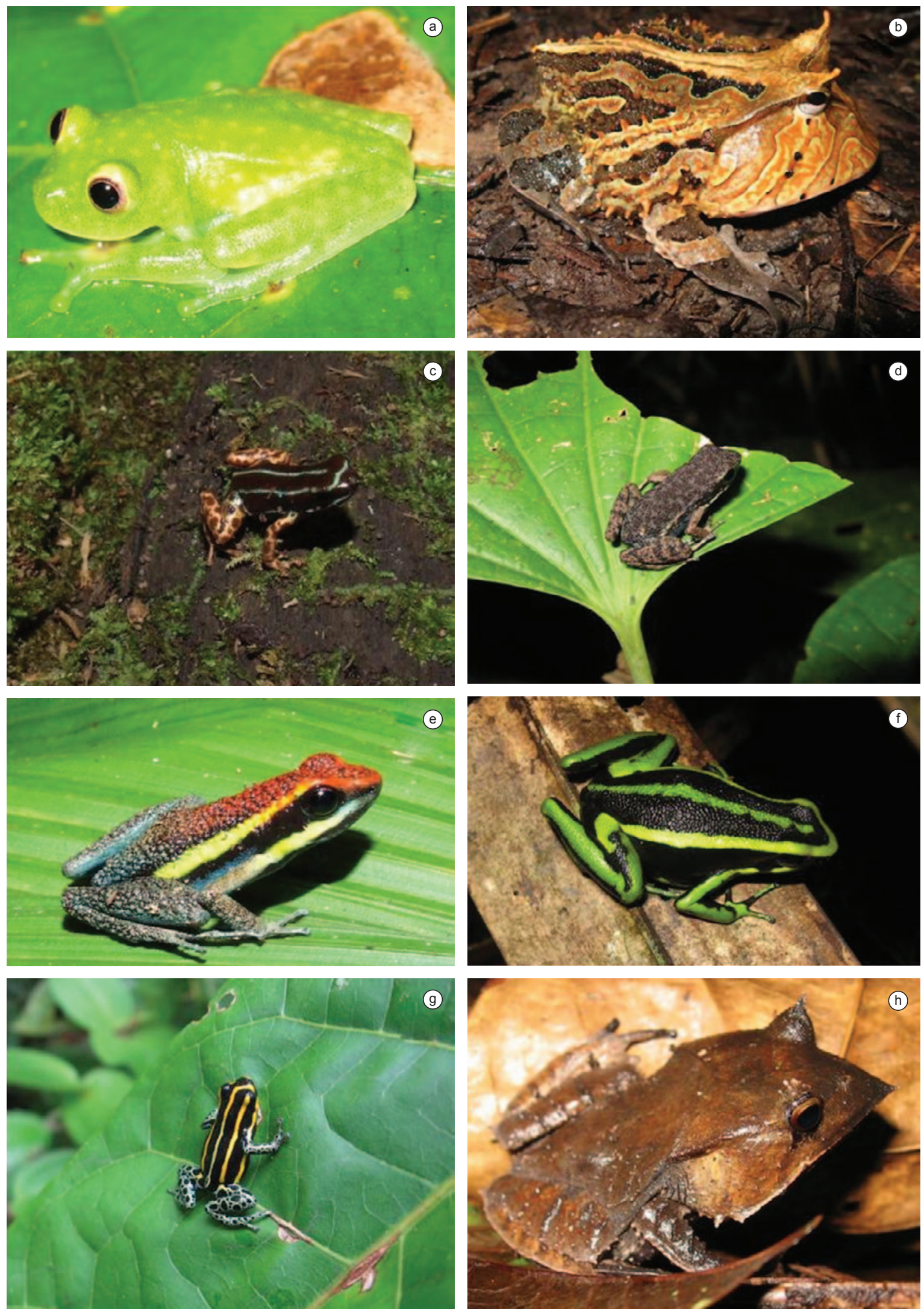

Figura 3. Algumas espécies registradas na Resex Riozinho da Liberdade (AC). a) Hyalinobatrachium munozorum; b) Ceratophys cornuta; c) Adelphobates sp.n.; d) Ameerega hahneli; e) Ameerega macero; f) Ameerega trivittata; g) Ranitomeya ventrimaculata; h) Hemiphractus scutatus.

Figure 3. Some species recorded in the Resex Riozinho da Liberdade (AC). a) Hyalinobatrachium munozorum; b) Ceratophys cornuta; c) Adelphobates sp.n.; d) Ameerega hahneli; e) Ameerega macero; f) Ameerega trivittata; g) Ranitomeya ventrimaculata; h) Hemiphractus scutatus. 

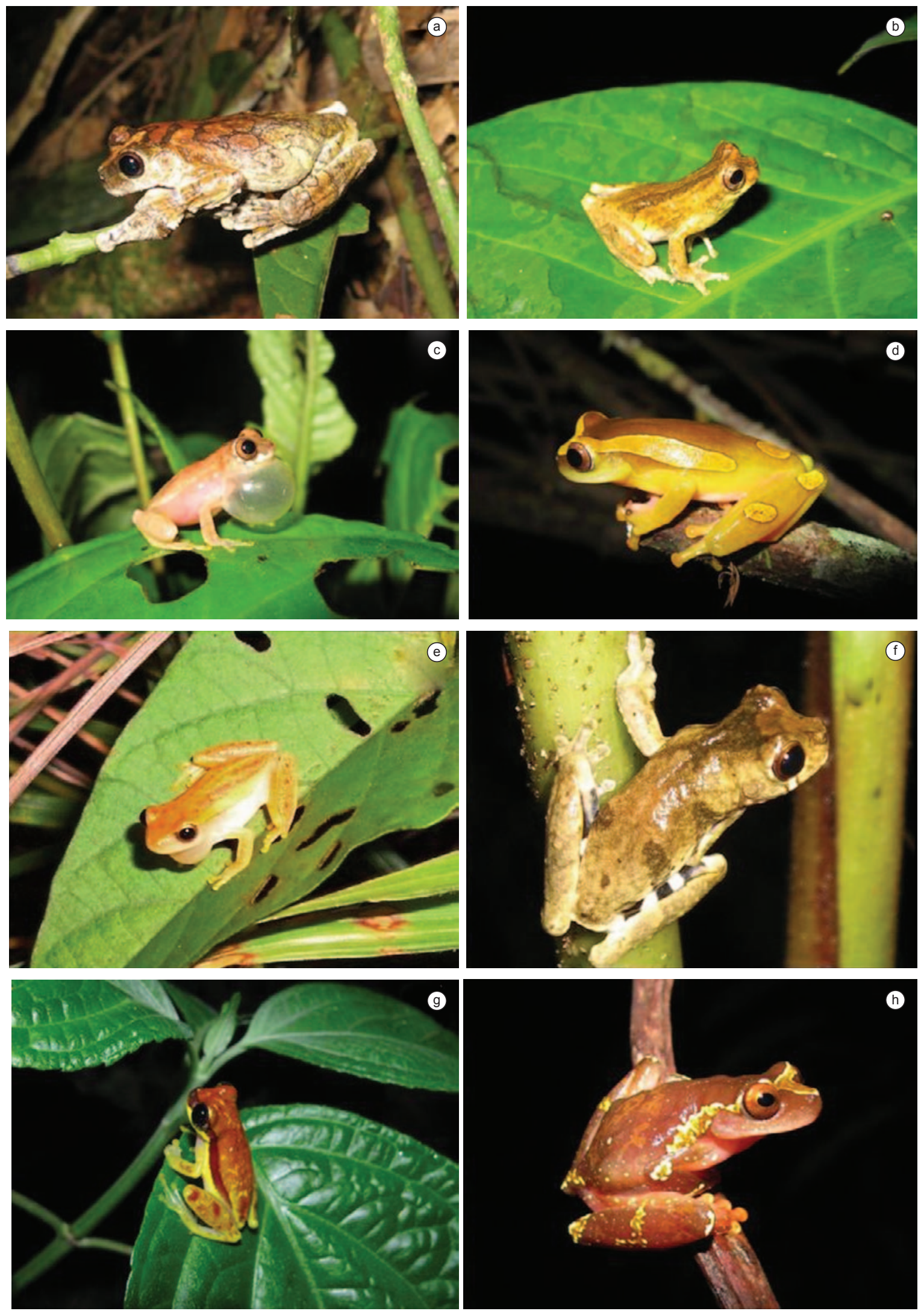

Figura 4. Algumas espécies registradas na Resex Riozinho da Liberdade (AC). a) Dendropsophus acreanus; b) Dendropsophus brevifrons; c) Dendropsophus leali; d) Dendropsophus leucophyllatus; e) Dendropsophus nanus; f) Dendropsophus parviceps; g) Dendropsophus rhodopeplus; h) Dendropsophus sarayacuensis.

Figure 4. Some species recorded in the Resex Riozinho da Liberdade (AC). a) Dendropsophus acreanus; b) Dendropsophus brevifrons; c) Dendropsophus leali; d) Dendropsophus leucophyllatus; e) Dendropsophus nanus; f) Dendropsophus parviceps; g) Dendropsophus rhodopeplus; h) Dendropsophus sarayacuensis. 

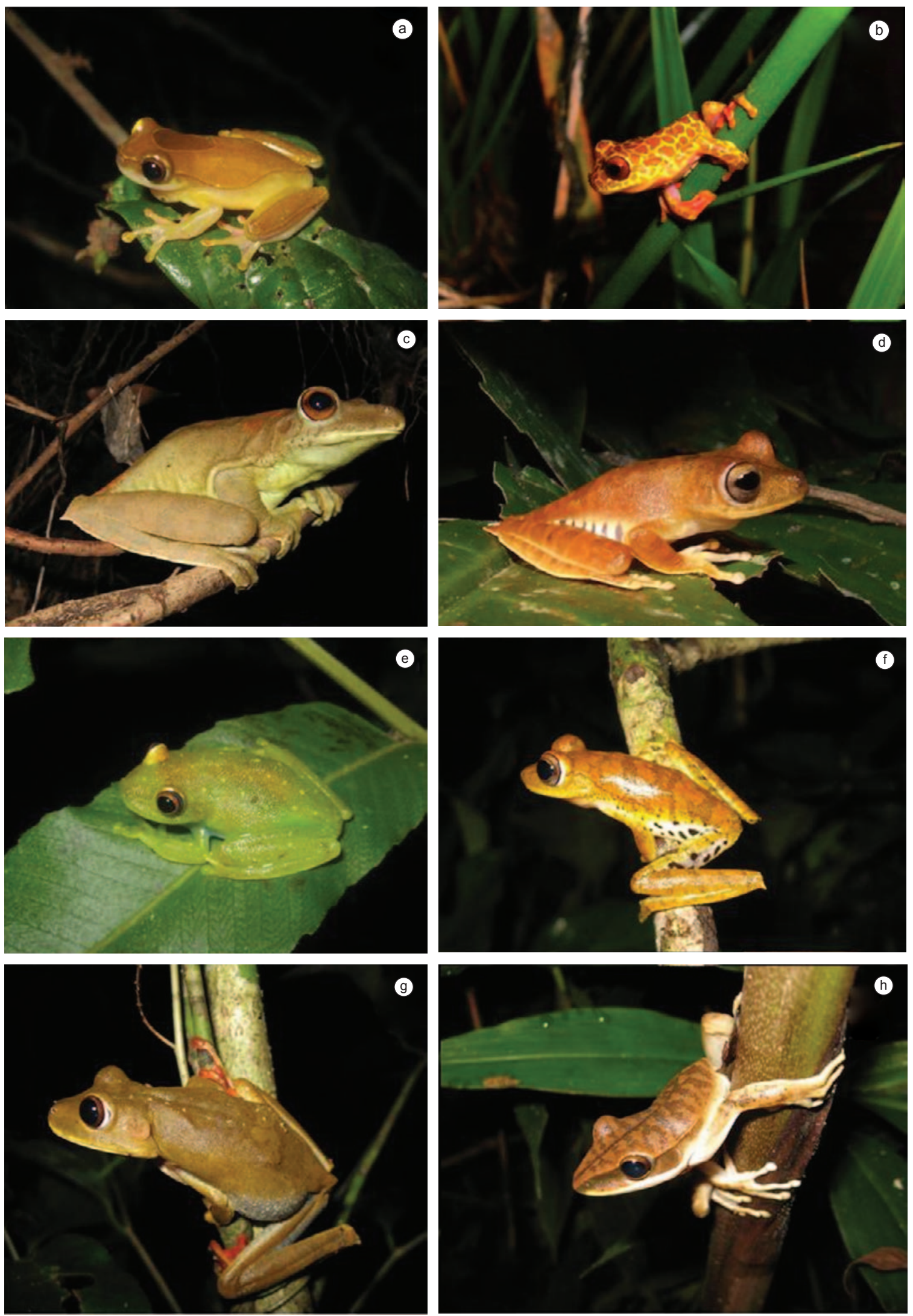

Figura 5. Algumas espécies registradas na Resex Riozinho da Liberdade (AC). a) Dendropsophus triangulum; b) Dendropsophus triangulum (padrão reticulado); c) Hypsiboas boans; d) Hypsiboas calcaratus; e) Hypsiboas cinerascens; f) Hypsiboas fasciatus; g) Hypsiboas geographicus; h) Hypsiboas lanciformis.

Figure 5. Some species recorded in the Resex Riozinho da Liberdade (AC). a) Dendropsophus triangulum; b) Dendropsophus triangulum (reticulated pattern); c) Hypsiboas boans; d) Hypsiboas calcaratus; e) Hypsiboas cinerascens; f) Hypsiboas fasciatus; g) Hypsiboas geographicus; h) Hypsiboas lanciformis. 
Bernarde, P.S. et al.
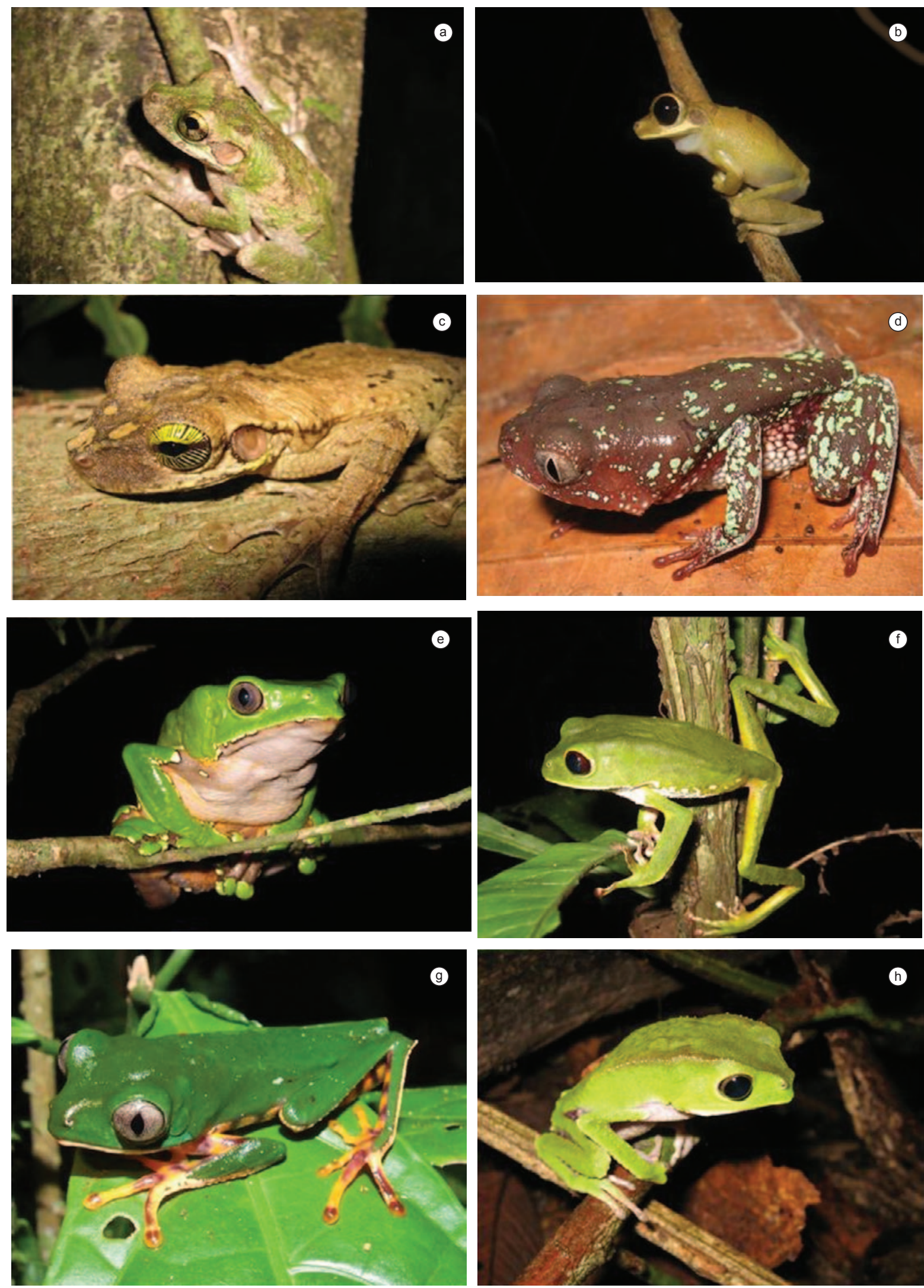

Figura 6. Algumas espécies registradas na Resex Riozinho da Liberdade (AC). a) Osteocephalus buckleyi; b) Osteocephalus leprieuri; c) Osteocephalus taurinus; d) Phyllomedusa atelopoides; e) Phyllomedusa bicolor; f) Phyllomedusa tarsius; g) Phyllomedusa tomopterna; h) Phyllomedusa vaillantii.

Figure 6. Some species recorded in the Resex Riozinho da Liberdade (AC). a) Osteocephalus buckleyi; b) Osteocephalus leprieuri; c) Osteocephalus taurinus; d) Phyllomedusa atelopoides; e) Phyllomedusa bicolor; f) Phyllomedusa tarsius; g) Phyllomedusa tomopterna; h) Phyllomedusa vaillantii. 

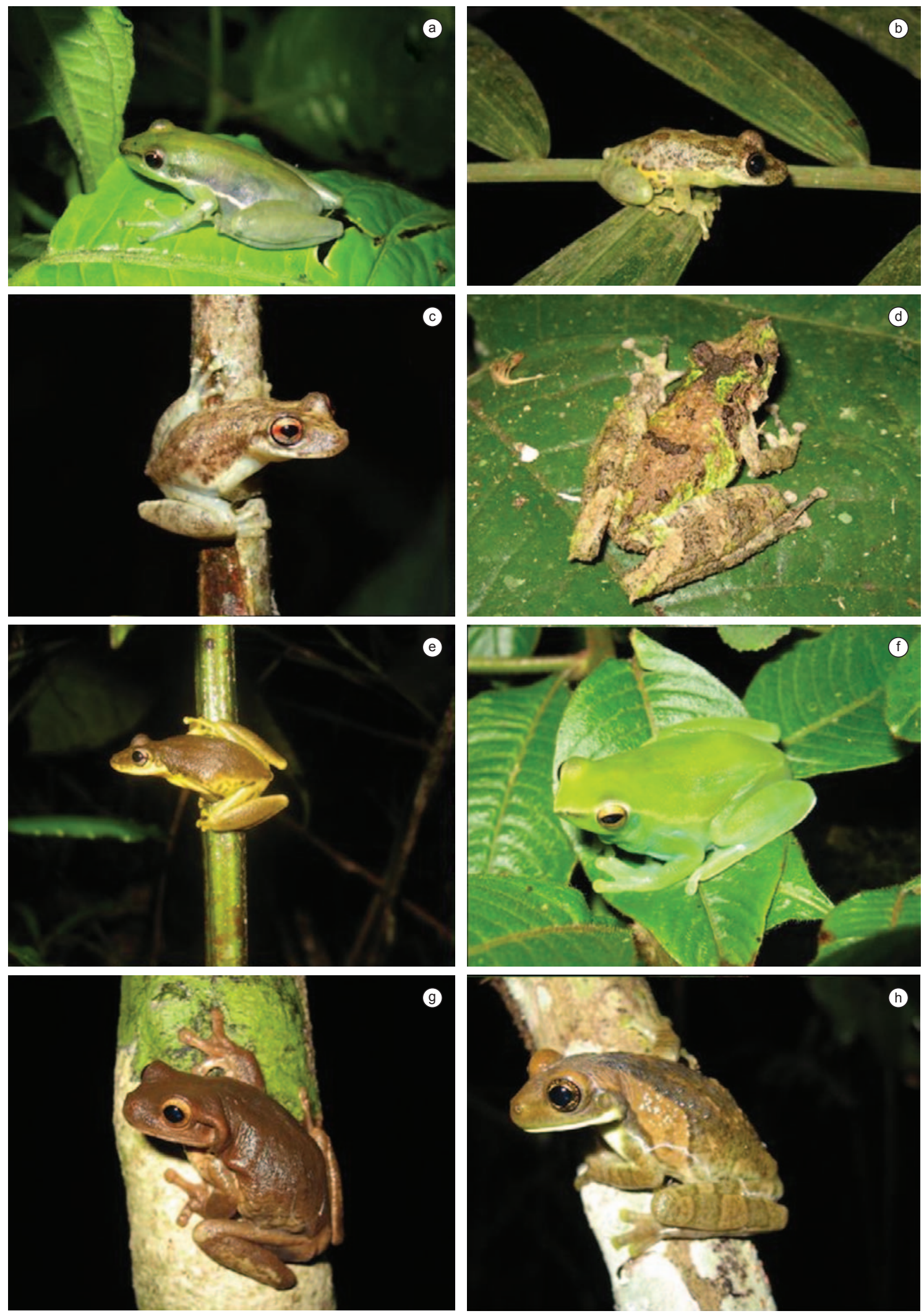

Figura 7. Algumas espécies registradas na Resex Riozinho da Liberdade (AC). a) Scarthyla goinorum; b) Scinax cruentommus; c) Scinax funereus; d) Scinax garbei; e) Scinax ruber; f) Sphaenorhynchus lacteus; g) Trachycephalus coriaceus; h) Trachycephalus typhonius (Linnaeus, 1758).

Figure 7. Some species recorded in the Resex Riozinho da Liberdade (AC). a) Scarthyla goinorum; b) Scinax cruentommus; c) Scinax funereus; d) Scinax garbei; e) Scinax ruber; f) Sphaenorhynchus lacteus; g) Trachycephalus coriaceus; h) Trachycephalus typhonius (Linnaeus, 1758). 

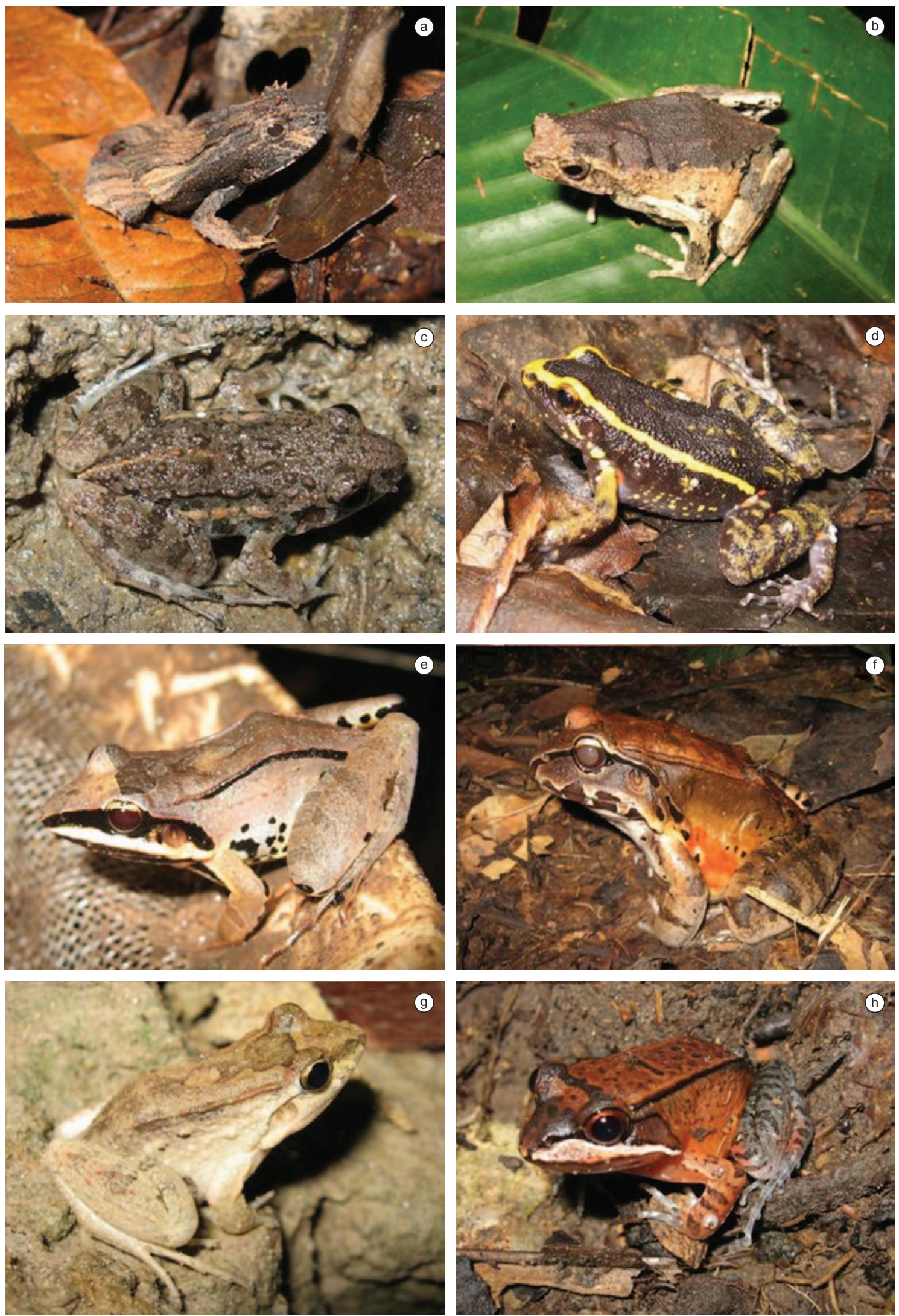

Figura 8. Algumas espécies registradas na Resex Riozinho da Liberdade (AC). a) Edalorhina perezi; b) Engystomops freibergi; c) Leptodactylus andreae; d) Leptodactylus lineatus; e) Leptodactylus mystaceus; f) Leptodactylus pentadactylus; g) Leptodactylus petersii; h) Leptodactylus rhodomystax.

Figure 8. Some species recorded in the Resex Riozinho da Liberdade (AC). a) Edalorhina perezi; b) Engystomops freibergi; c) Leptodactylus andreae; d) Leptodactylus lineatus; e) Leptodactylus mystaceus; f) Leptodactylus pentadactylus; g) Leptodactylus petersii; h) Leptodactylus rhodomystax. 

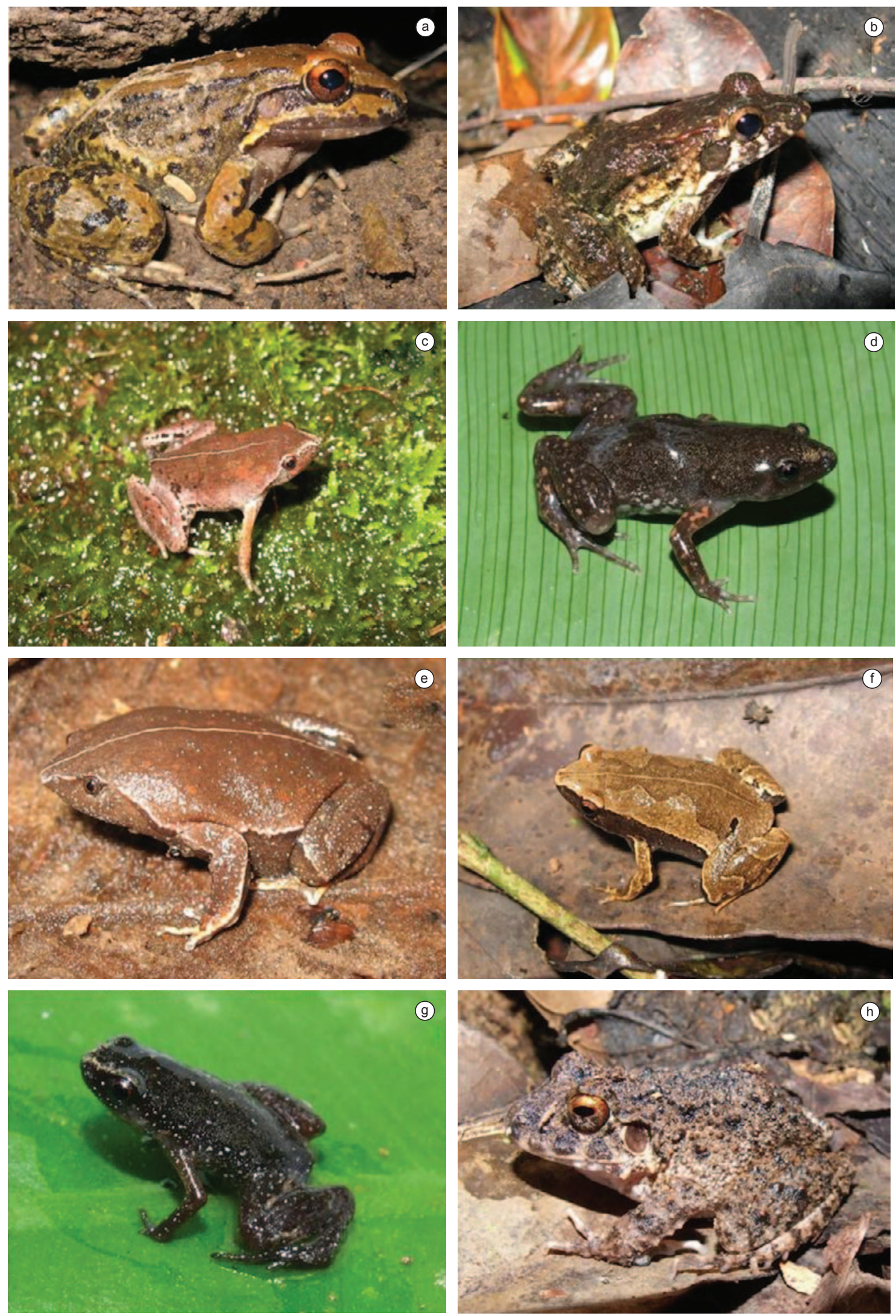

Figura 9. Algumas espécies registradas na Resex Riozinho da Liberdade (AC). a) Leptodactylus rhodonotus; b) Leptodactylus wagneri; c) Chiasmocleis bassleri; d) Chiasmocleis ventrimaculata; e) Ctenophryne geayi; f) Hamptophryne boliviana; g) Syncope antenori; h) Oreobates quixensis.

Figure 9. Some species recorded in the Resex Riozinho da Liberdade (AC). a) Leptodactylus rhodonotus; b) Leptodactylus wagneri; c) Chiasmocleis bassleri; d) Chiasmocleis ventrimaculata; e) Ctenophryne geayi; f) Hamptophryne boliviana; g) Syncope antenori; h) Oreobates quixensis. 
Bernarde, P.S. et al.
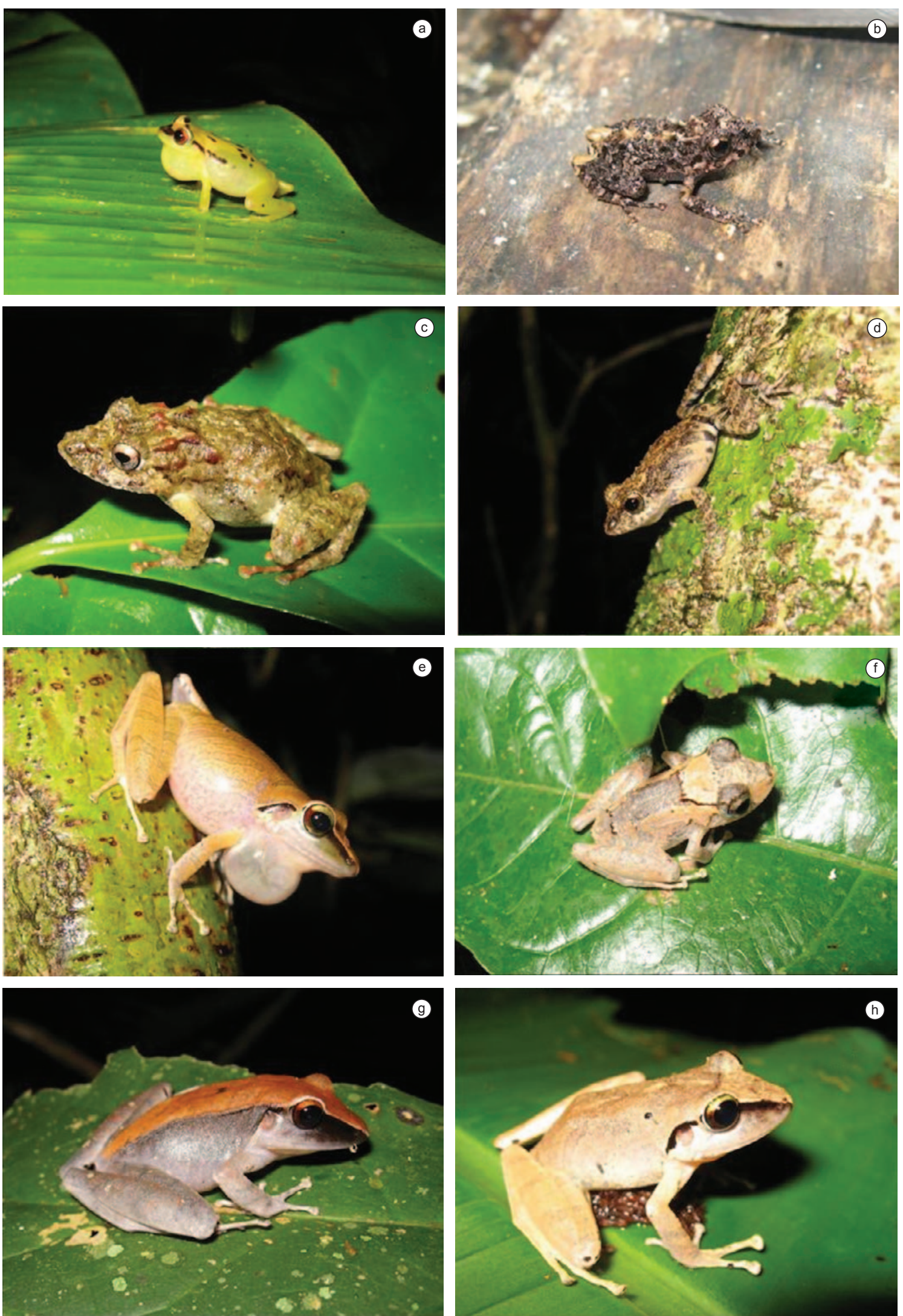

Figura 10. Algumas espécies registradas na Resex Riozinho da Liberdade (AC). a) Pristimantis acuminatus; b) Pristimantis altamazonicus; c) Pristimantis academicus; d) Pristimantis diadematus; e) Pristimantis fenestratus; f) Pristimantis ockendeni; g) Pristimantis peruvianus; h) Pristimantis reichlei.

Figure 10. Some species recorded in the Resex Riozinho da Liberdade (AC). a) Pristimantis acuminatus; b) Pristimantis altamazonicus; c) Pristimantis academicus; d) Pristimantis diadematus; e) Pristimantis fenestratus; f) Pristimantis ockendeni; g) Pristimantis peruvianus; h) Pristimantis reichlei. 

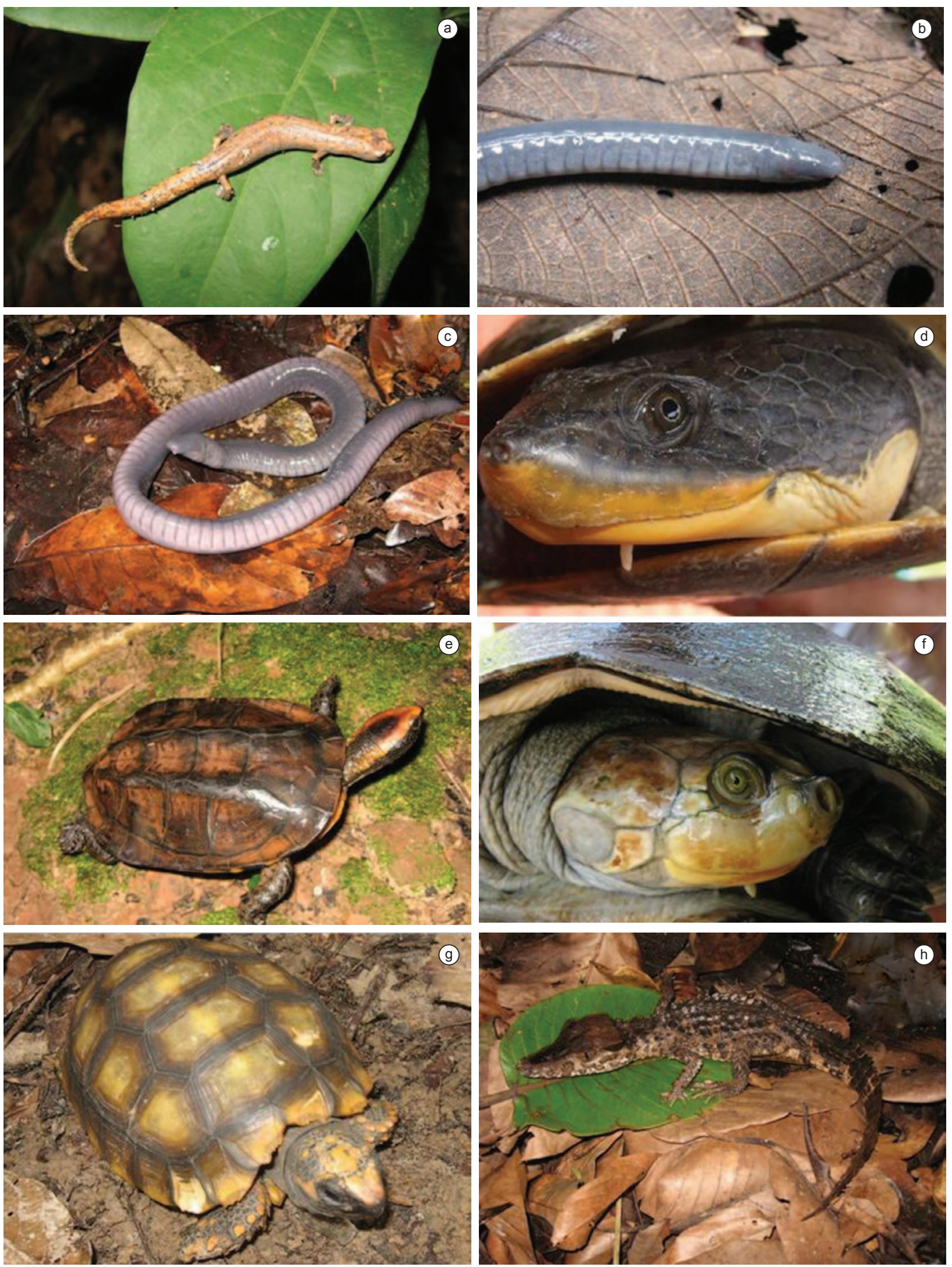

Figura 11. Algumas espécies registradas na Resex Riozinho da Liberdade (AC). a) Bolitoglossa sp.; b) Caecilia marcusi; c) Caecilia tentaculata; d) Mesoclemmys gibba; e) Platemys platycephala; f) Podocnemis unifilis; g) Chelonoidis denticulata; h) Paleosuchus palpebrosus.

Figure 11. Some species recorded in the Resex Riozinho da Liberdade (AC). a) Bolitoglossa sp.; b) Caecilia marcusi; c) Caecilia tentaculata; d) Mesoclemmys gibba; e) Platemys platycephala; f) Podocnemis unifilis; g) Chelonoidis denticulata; h) Paleosuchus palpebrosus. 
Bernarde, P.S. et al.
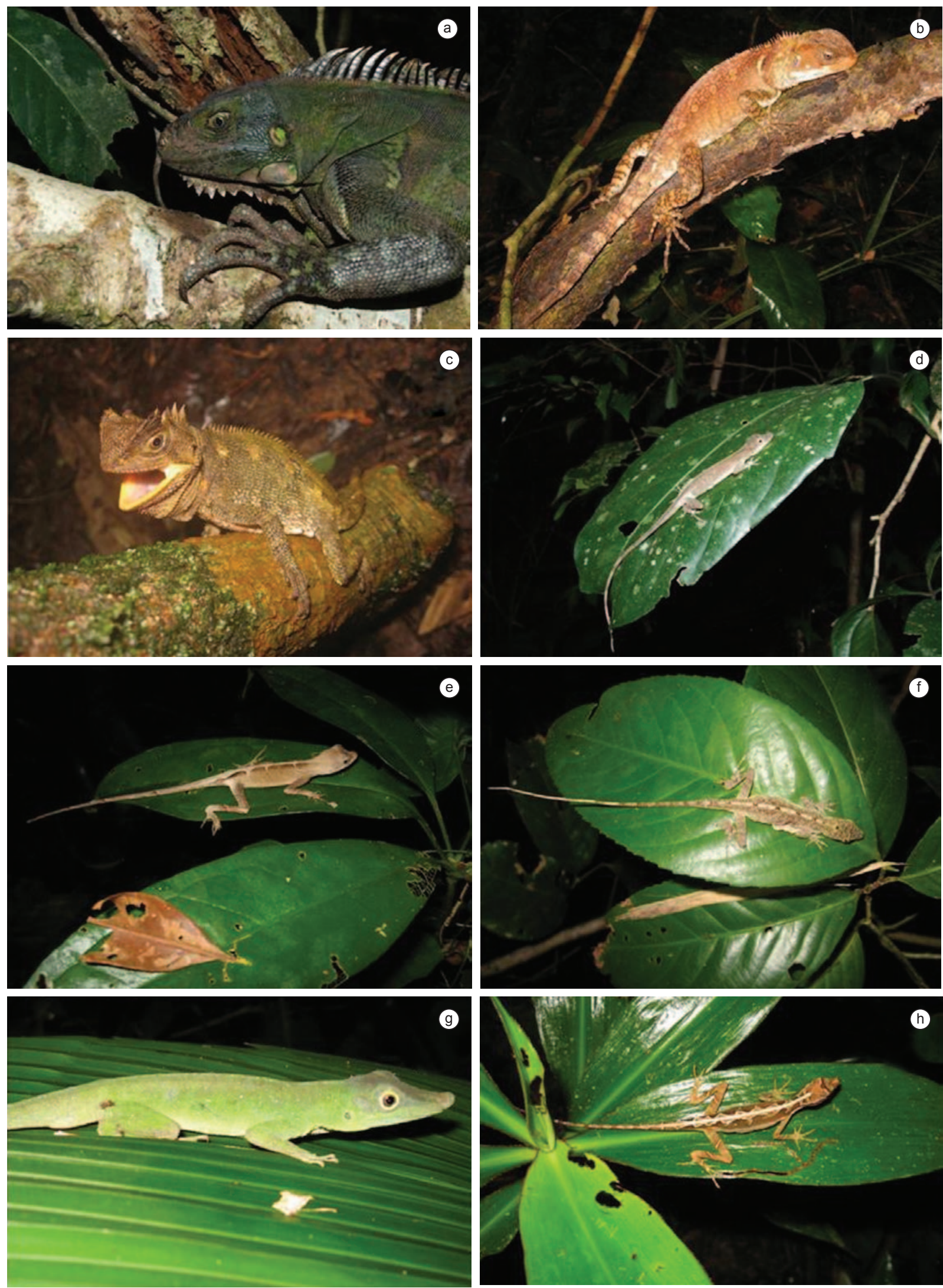

Figura 12. Algumas espécies registradas na Resex Riozinho da Liberdade (AC). a) Iguana iguana; b) Enyalioides laticeps; c) Enyalioides palpebralis; d) Anolis fuscoauratus; e) Anolis nitens; f) Anolis ortonii; g) Anolis punctatus; h) Anolis trachyderma.

Figure 12. Some species recorded in the Resex Riozinho da Liberdade (AC). a) Iguana iguana; b) Enyalioides laticeps; c) Enyalioides palpebralis; d) Anolis fuscoauratus; e) Anolis nitens; f) Anolis ortonii; g) Anolis punctatus; h) Anolis trachyderma. 

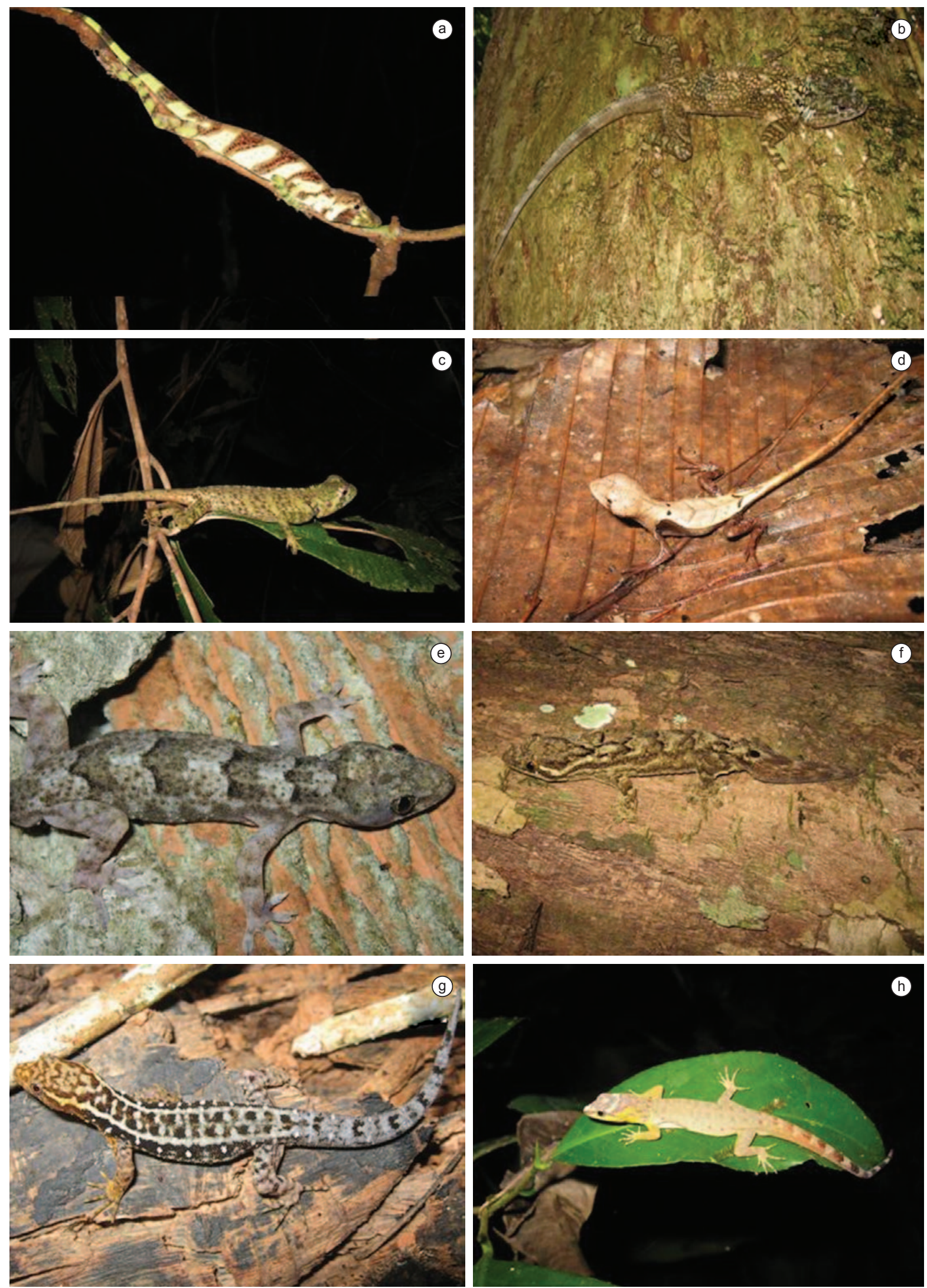

Figura 13. Algumas espécies registradas na Resex Riozinho da Liberdade (AC). a) Anolis transversalis; b) Plica plica; c) Plica umbra; d) Stenocercus fimbriatus; e) Hemidactylus mabouia; f) Thecadactylus solimoensis; g) Gonatodes hasemani; h) Gonatodes humeralis.

Figure 13. Some species recorded in the Resex Riozinho da Liberdade (AC). a) Anolis transversalis; b) Plica plica; c) Plica umbra; d) Stenocercus fimbriatus; e) Hemidactylus mabouia; f) Thecadactylus solimoensis; g) Gonatodes hasemani; h) Gonatodes humeralis. 
Bernarde, P.S. et al.
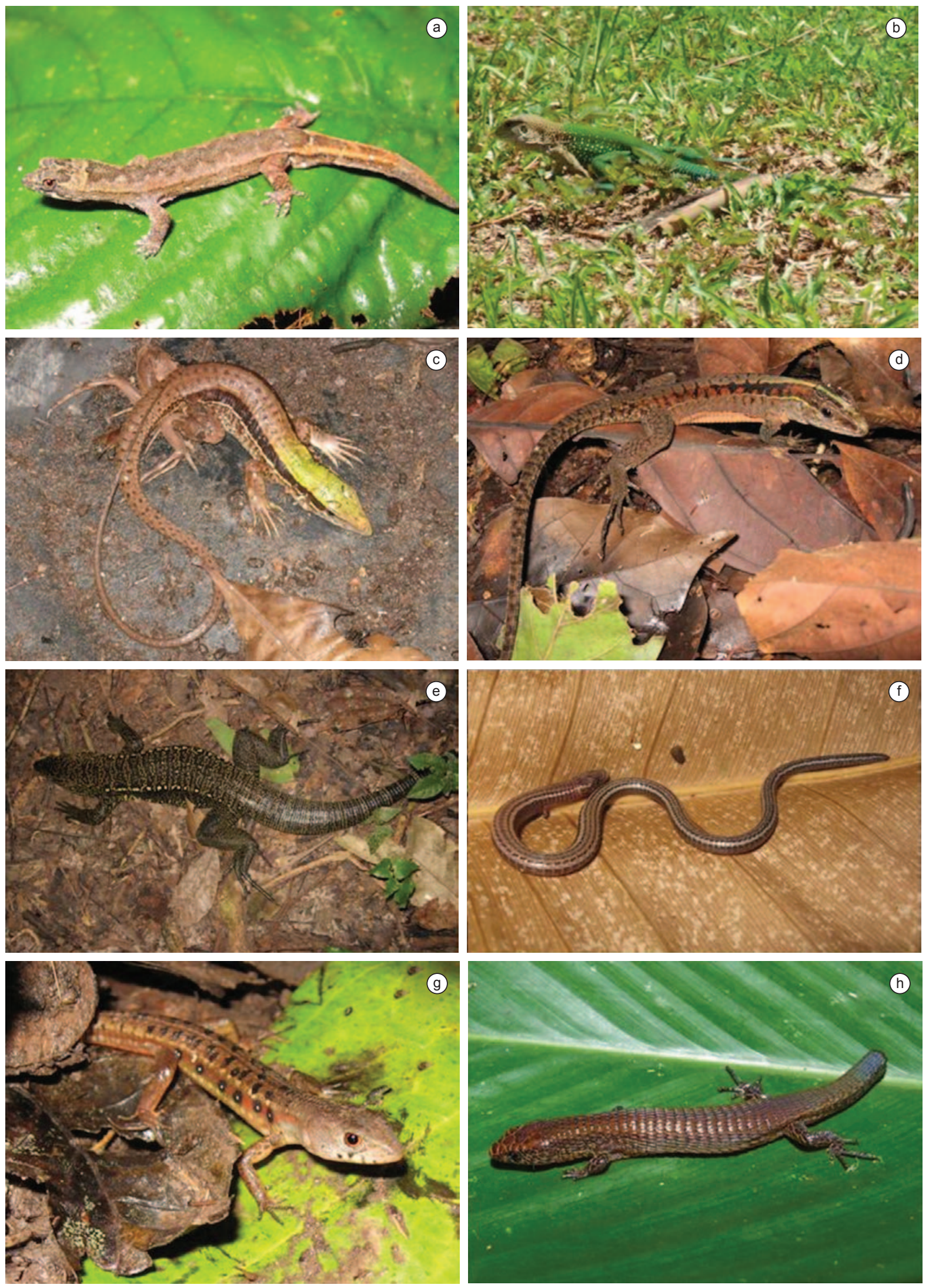

Figura 14. Algumas espécies registradas na Resex Riozinho da Liberdade (AC). a) Pseudogonatodes guianensis; b) Ameiva ameiva; c) Kentropyx altamazonica; d) Kentropyx pelviceps; e) Tupinambis teguixin; f) Bachia peruana; g) Cercosaura ocellata; h) Ptychoglossus brevifrontalis.

Figure 14. Some species recorded in the Resex Riozinho da Liberdade (AC). a) Pseudogonatodes guianensis; b) Ameiva ameiva; c) Kentropyx altamazonica; d) Kentropyx pelviceps; e) Tupinambis teguixin; f) Bachia peruana; g) Cercosaura ocellata; h) Ptychoglossus brevifrontalis. 

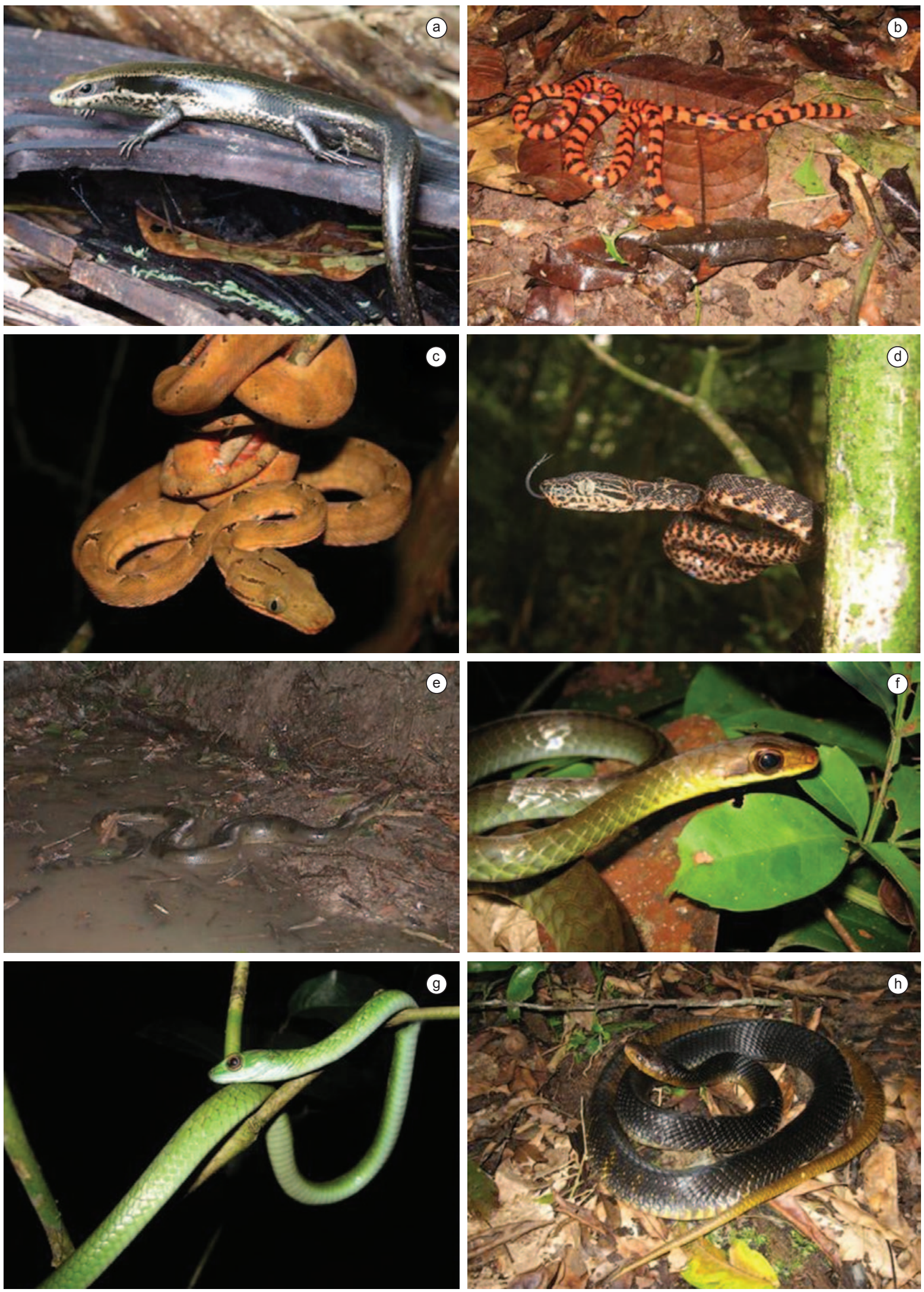

Figura 15. Algumas espécies registradas na Resex Riozinho da Liberdade (AC). a) Mabuya nigropunctata; b) Anilius scytale; c) Corallus hortulanus; d) Corallus hortulanus; e) Eunectes murinus; f) Chironius carinatus; g) Chironius scurrulus (padrão juvenil); h) Drymarchon corais.

Figure 15. Some species recorded in the Resex Riozinho da Liberdade (AC). a) Mabuya nigropunctata; b) Anilius scytale; c) Corallus hortulanus; d) Corallus hortulanus; e) Eunectes murinus; f) Chironius carinatus; g) Chironius scurrulus (juvenile pattern); h) Drymarchon corais. 
Bernarde, P.S. et al.
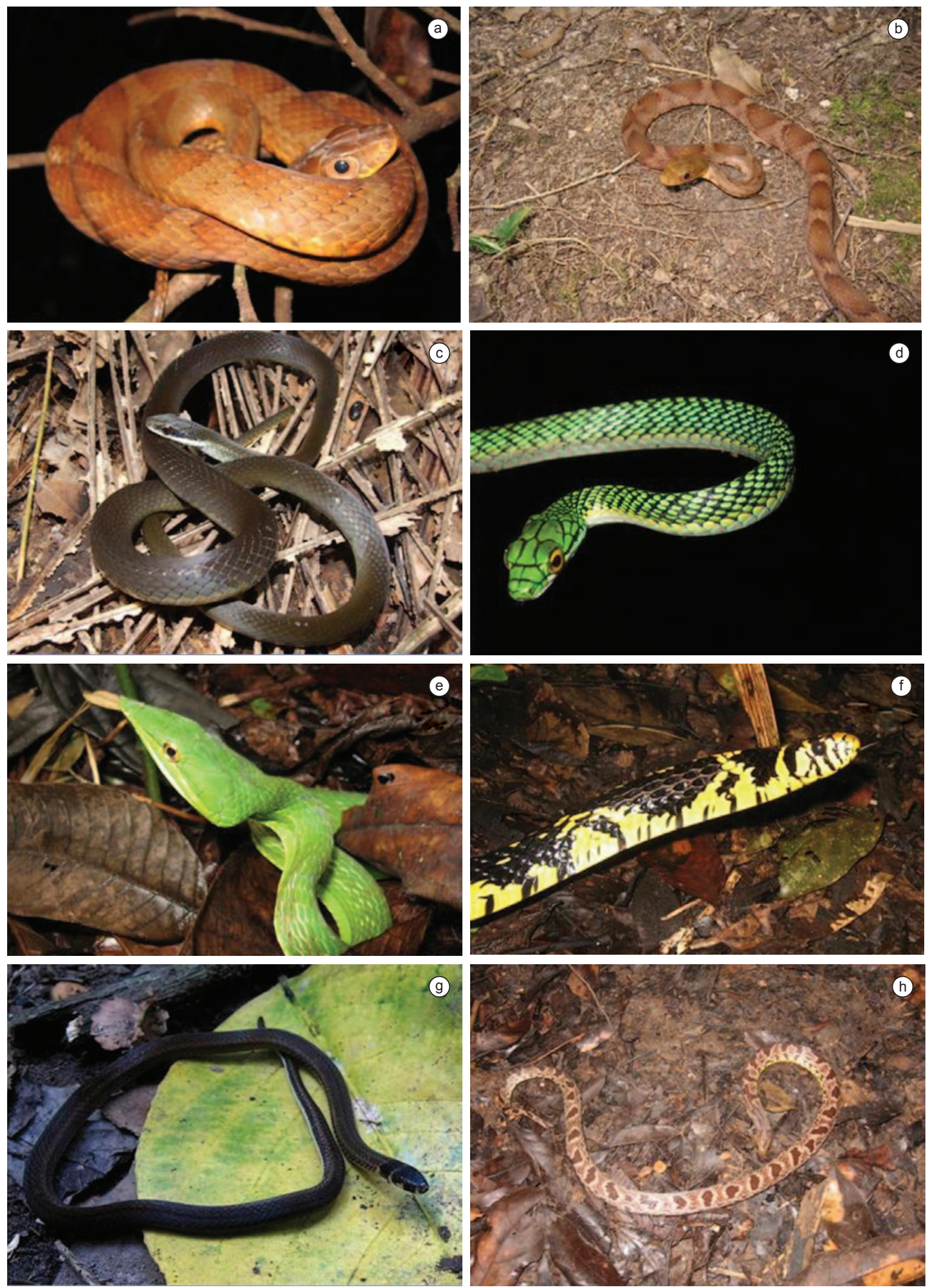

Figura 16. Algumas espécies registradas na Resex Riozinho da Liberdade (AC). a) Drymobius rhombifer; b) Drymobius rhombifer; c) Drymoluber dichrous; d) Leptophis ahaetulla; e) Oxybelis fulgidus; f) Spilotes pullatus; g) Tantilla melanocephala; h) Atractus major.

Figure 16. Some species recorded in the Resex Riozinho da Liberdade (AC). a) Drymobius rhombifer; b) Drymobius rhombifer; c) Drymoluber dichrous; d) Leptophis ahaetulla; e) Oxybelis fulgidus; f) Spilotes pullatus; g) Tantilla melanocephala; h) Atractus major. 

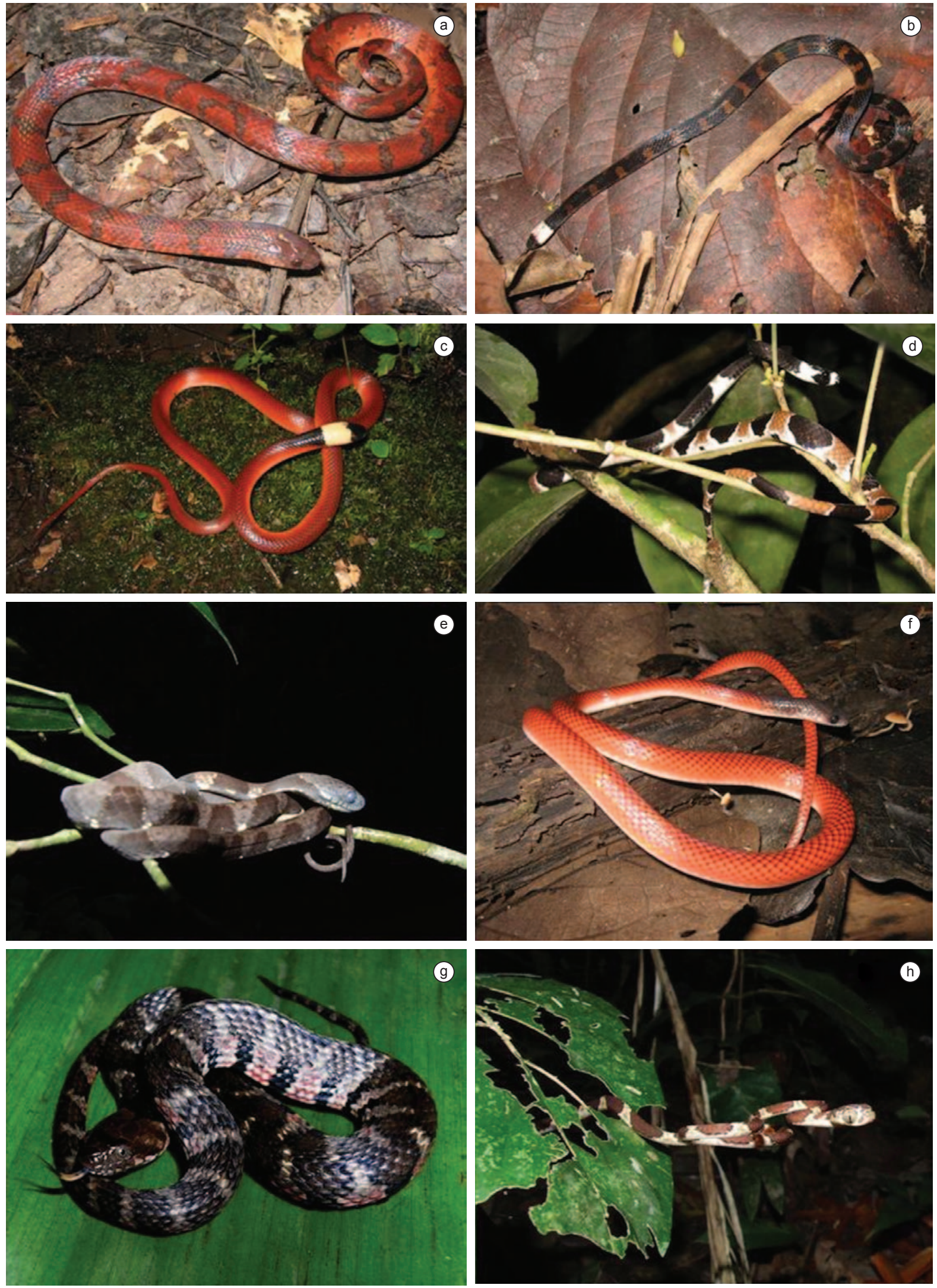

Figura 17. Algumas espécies registradas na Resex Riozinho da Liberdade (AC). a) Atractus major; b) Atractus schach (padrão juvenil); c) Clelia clelia (padrão juvenil); d) Dipsas catesbyi; e) Dipsas indica; f) Drepanoides anomalus; g) Helicops angulatus; h) Imantodes cenchoa.

Figure 17. Some species recorded in the Resex Riozinho da Liberdade (AC). a) Atractus major; b) Atractus schach (juvenile pattern); c) Clelia clelia (juvenile pattern); d) Dipsas catesbyi; e) Dipsas indica; f) Drepanoides anomalus; g) Helicops angulatus; h) Imantodes cenchoa. 

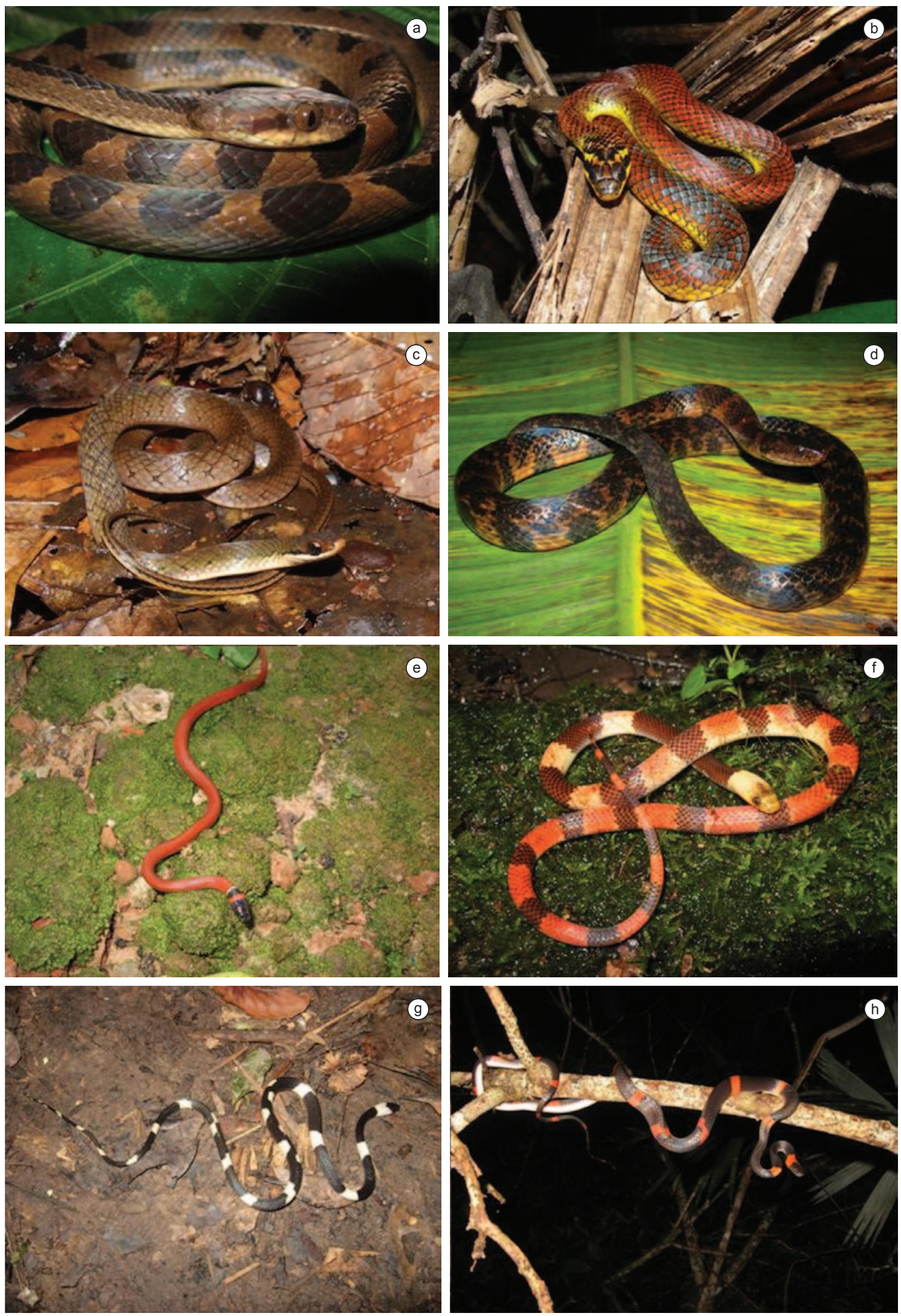

Figura 18. Algumas espécies registradas na Resex Riozinho da Liberdade (AC). a) Leptodeira annulata; b) Liophis dorsocorallinus; c) Liophis reginae; d) Liophis taeniogaster; e) Oxyrhopus melanogenys; f) Oxyrhopus occipitalis; g) Oxyrhophus petola (padrão juvenil); h) Oxyrhopus petola.

Figure 18. Some species recorded in the Resex Riozinho da Liberdade (AC). a) Leptodeira annulata; b) Liophis dorsocorallinus; c) Liophis reginae; d) Liophis taeniogaster; e) Oxyrhopus melanogenys; f) Oxyrhopus occipitalis; g) Oxyrhophus petola (juvenile pattern); h) Oxyrhopus petola. 

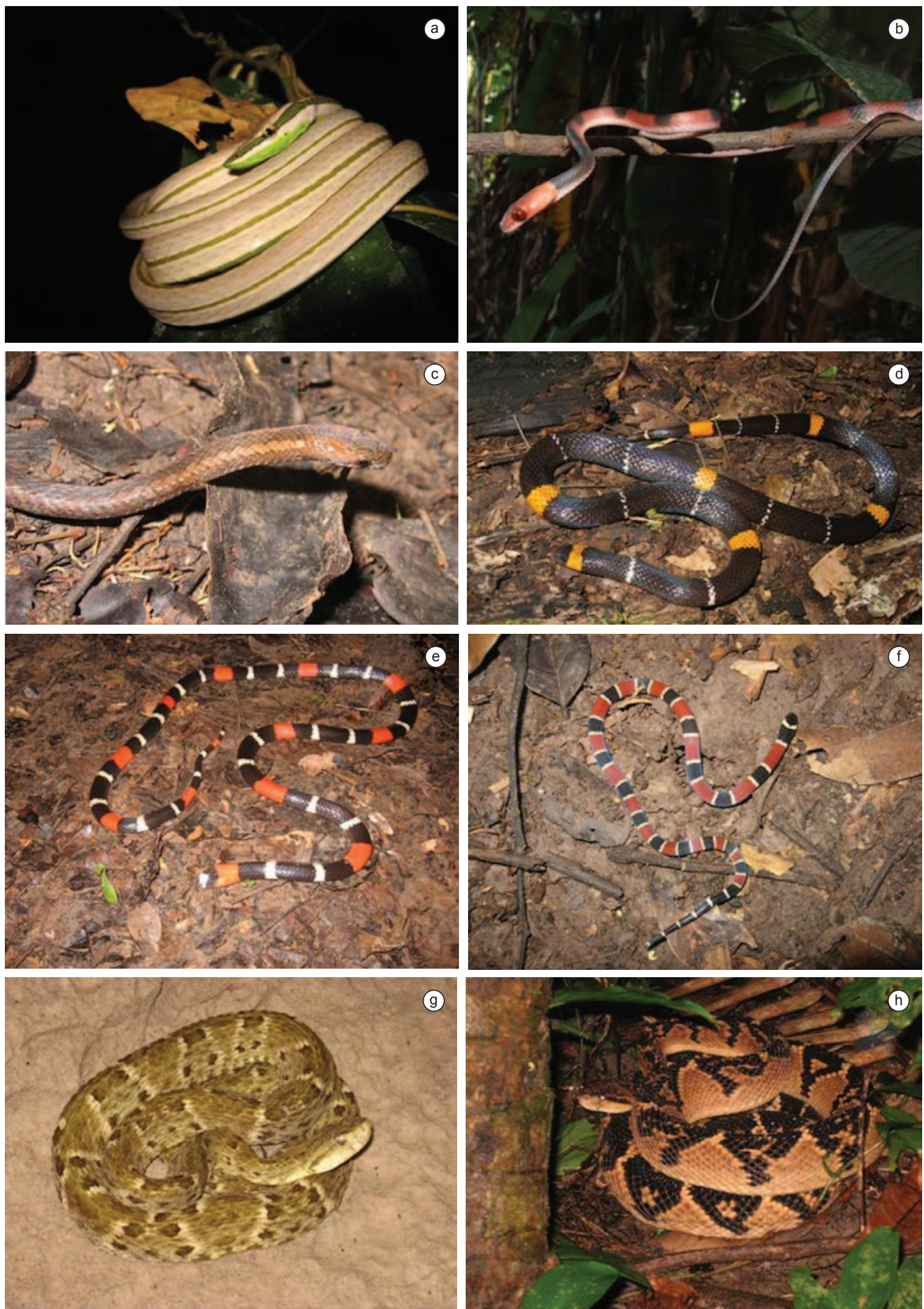

Figura 19. Algumas espécies registradas na Resex Riozinho da Liberdade (AC). a) Philodryas argentea; b) Siphlophis compressus; c) Taeniophallus brevirostris; d) Micrurus hemprichii; e) Micrurus lemniscatus; f) Micrurus remotus; g) Bothrops atrox; h) Lachesis muta.

Figure 19. Some species recorded in the Resex Riozinho da Liberdade (AC). a) Philodryas argentea; b) Siphlophis compressus; c) Taeniophallus brevirostris; d) Micrurus hemprichii; e) Micrurus lemniscatus; f) Micrurus remotus; g) Bothrops atrox; h) Lachesis muta. 

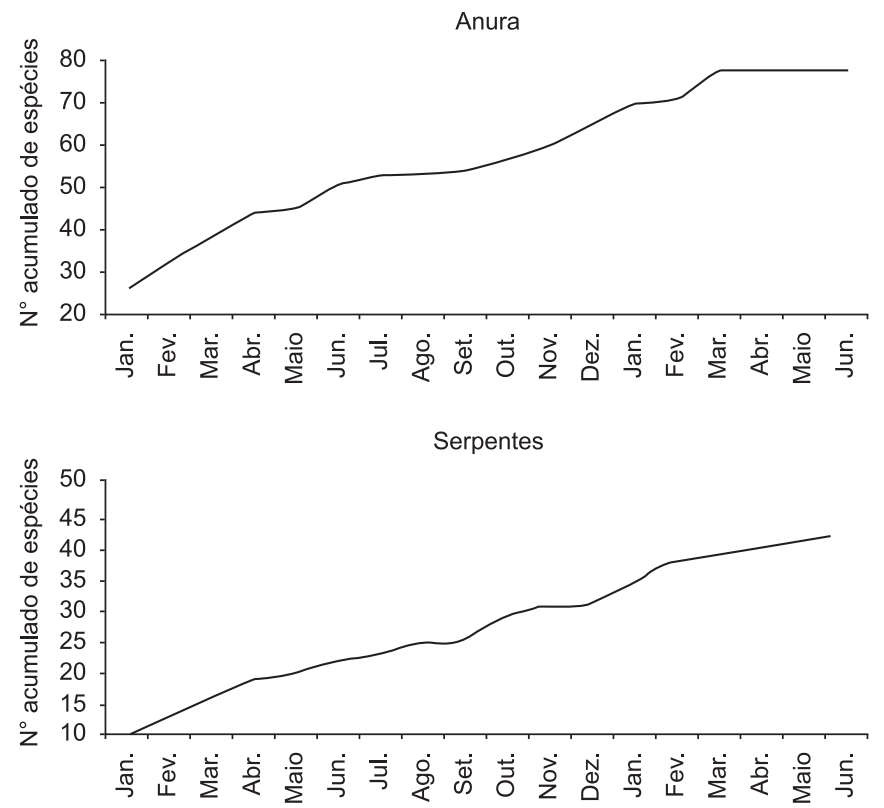
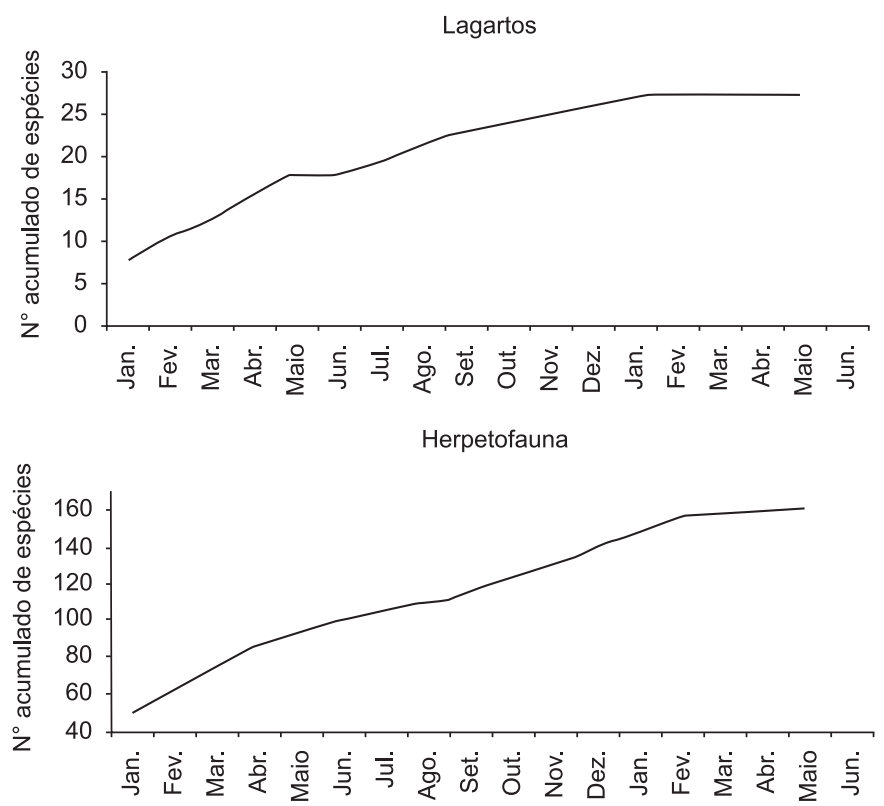

Figura 20. Curva acumulada de espécies para anuros, lagartos, serpentes e herpetofauna total na Resex Riozinho da Liberdade (AC).

Figure 20. Acumulated curve of species from frogs, lizards, snakes and total herpetofauna in the Resex Riozinho da Liberdade (AC).

Lista das espécies da herpetofauna registrada na Resex Riozinho da Liberdade - AC.

\section{AMPHIBIA}

\section{ANURA}

Aromobatidae

1. Allobates femoralis (Boulenger, 1884)

2. Allobates sp. 1

3. Allobates $\mathrm{sp} .2$

Bufonidae

4. Dendrophryniscus minutus (Peters, 1872)

5. Rhaebo guttatus (Schneider, 1799)

6. Rhinella castaneotica (Caldwell, 1991)

7. Rhinella margaritifera (Laurenti, 1768)

8. Rhinella marina (Linnaeus, 1758)

9. Rhinella sp.

Centrolenidae

10. Hyalinobatrachium munozorum (Lynch \& Duellman, 1973)

Ceratophryidae

11. Ceratophrys cornuta (Linnaeus, 1758)

Dendrobatidae

12. Adelphobates quinquevittatus (Steindachner, 1864)

13. Adelphobates sp.n.

14. Ameerega hahneli (Boulenger, 1884)

15. Ameerega macero (Rodriguéz \& Myers, 1993)

16. Ameerega trivittata (Spix, 1824)

17. Ranitomeya ventrimaculata (Shreve, 1935)

Hemiphractidae

18. Hemiphractus scutatus (Spix, 1824)

Hylidae

19. Dendropsophus acreanus (Bokermann, 1964)
20. Dendropsophus bokermanni (Goin, 1960)

21. Dendropsophus brevifrons (Duellman \& Crump, 1974)

22. Dendropsophus leali (Bokermann, 1964)

23. Dendropsophus leucophyllatus (Beireis, 1783)

24. Dendropsophus nanus (Boulenger, 1889)

25. Dendropsophus parviceps (Boulenger, 1882)

26. Dendropsophus rhodopeplus (Günther, 1859)

27. Dendropsophus sarayacuensis (Shreve, 1935)

28. Dendropsophus triangulum (Günther, 1869)

29. Hypsiboas boans (Linnaeus, 1758)

30. Hypsiboas calcaratus (Günther, 1858)

31. Hypsiboas cinerascens (Spix, 1824)

32. Hypsiboas fasciatus (Günther, 1858)

33. Hypsiboas geographicus (Spix, 1824)

34. Hypsiboas lanciformis (Cope, 1871)

35. Osteocephalus buckleyi (Boulenger, 1882)

36. Osteocephalus leprieuri (Duméril \& Bibron, 1841)

37. Osteocephalus taurinus Steindachner, 1862

38. Osteocephalus sp.

39. Phyllomedusa atelopoides Duellman, Cadle \& Cannatella, 1988

40. Phyllomedusa bicolor (Boddaert, 1772)

41. Phyllomedusa tarsius (Cope, 1868)

42. Phyllomedusa tomopterna (Cope, 1868)

43. Phyllomedusa vaillantii Boulenger, 1882

44. Scarthyla goinorum (Bokermann, 1962)

45. Scinax boesemani (Goin, 1966)

46. Scinax cruentommus (Duellman, 1972)

47. Scinax funereus (Cope, 1874)

48. Scinax garbei (Miranda-Ribeiro, 1926)

49. Scinax ruber (Laurenti, 1768)

50. Sphaenorhynchus dorisae (Goin, 1957)

51. Sphaenorhynchus lacteus (Daudin, 1801)

52. Trachycephalus coriaceus (Peters, 1867)

53. Trachycephalus resinifictrix (Goeldi, 1907)

54. Trachycephalus typhonius (Linnaeus, 1758) 
Leiuperidae

55. Edalorhina perezi Jiménez de la Espada, 1871

56. Engystomops freibergi (Donoso-Barros, 1969)

Leptodactylidae

57. Leptodactylus andreae (Müller, 1923)

58. Leptodactylus lineatus (Schneider, 1799)

59. Leptodactylus mystaceus (Spix, 1824)

60. Leptodactylus pentadactylus (Laurenti, 1768)

61. Leptodactylus petersii (Steindachner, 1864)

62. Leptodactylus rhodomystax Boulenger, 1884

63. Leptodactylus rhodonotus (Günther, 1869)

64. Leptodactylus wagneri (Peters, 1862)

Microhylidae

65. Chiasmocleis bassleri Dunn, 1949

66. Chiasmocleis ventrimaculata (Andersson, 1945)

67. Ctenophryne geayi Mocquard, 1904

68. Hamptophryne boliviana (Parker, 1927)

69. Syncope antenori Walker, 1973

Strabomantidae

70. Oreobates quixensis Jiménez de la Espada, 1872

71. Pristimantis academicus Lehr, Moravec \& Urrutia, 2010

72. Pristimantis acuminatus (Shreve, 1935)

73. Pristimantis altamazonicus (Barbour \& Dunn, 1921)

74. Pristimantis aureolineatus Guayasamin, Ron, Cisneros-

Heredia, Lamar \& McCracken, 2006

75. Pristimantis conspicillatus (Günther, 1858)

76. Pristimantis diadematus (Jiménez de la Espada, 1875)

77. Pristimantis fenestratus (Steindachner, 1864)

78. Pristimantis ockendeni (Boulenger, 1912)

79. Pristimantis peruvianus (Melin, 1941)

80. Pristimantis reichlei Padial \& De la Riva, 2009

\section{URODELA}

Plethodontidae

81. Bolitoglossa sp.

\section{GYMNOPHIONA}

Caeciliidae

82. Caecilia marcusi Wake, 1985

83. Caecilia tentaculata Linnaeus, 1758

\section{REPTILIA}

\section{TESTUDINES}

Chelidae

84. Mesoclemmys gibba (Schweigger, 1812)

85. Platemys platycephala (Schneider, 1792)

Podocnemididae

86. Podocnemis unifilis Troschel, 1848

Testudinidae

87. Chelonoidis denticulata (Linnaeus, 1766)

\section{CROCODYLIA}

Alligatoridae

88. Caiman crocodilus (Linnaeus, 1758)

89. Melanosuchus niger (Spix, 1825)

90. Paleosuchus palpebrosus (Cuvier, 1807)
SQUAMATA

AMPHISBAENIA

Amphisbaenidae

91. Amphisbaena fuliginosa Linnaeus, 1758

SAURIA

Iguanidae

92. Iguana iguana (Linnaeus, 1758)

Hoplocercidae

93. Enyalioides laticeps (Guichenot, 1855)

94. Enyalioides palpebralis (Boulenger, 1883)

Polychrotidae

95. Anolis fuscoauratus D’Orbigny, 1837

96 Anolis nitens (Wagler, 1830)

97. Anolis ortonii Cope, 1868

98. Anolis punctatus Daudin, 1802

99. Anolis trachyderma Cope, 1876

100. Anolis transversalis Duméril, 1851

Tropiduridae

101. Plica plica (Linnaeus, 1758)

102. Plica umbra (Linnaeus, 1758)

103. Stenocercus fimbriatus Avila-Pires, 1995

Gekkonidae

104. Hemidactylus mabouia (Moreau de Jonnès, 1818)

Phyllodactylidae

105. Thecadactylus solimoensis Bergmann \& Russell, 2007

Sphaerodactylidae

106. Gonatodes hasemani (Griffin, 1917)

107. Gonatodes humeralis (Guichenot, 1855)

108. Pseudogonatodes guianensis Parker, 1935

Teiidae

109. Ameiva ameiva (Linnaeus, 1758)

110. Dracaena guianensis Daundin, 1802

111. Kentropyx altamazonica Cope, 1876

112. Kentropyx pelviceps Cope, 1868

113. Tupinambis teguixin (Linnaeus, 1758)

Gymnophthalmidae

114. Alopoglossus buckleyi (O’Shaughnessy, 1881)

115. Bachia peruana (Werner, 1901)

116. Cercosaura argulus Peters, 1863

117. Cercosaura ocellata Wagler, 1830

118. Iphisa elegans Gray 1851

119. Ptychoglossus brevifrontalis Boulenger, 1912

Scincidae

120. Mabuya nigropunctata (Spix, 1825)

\section{SERPENTES}

ANILIIDAE

121. Anilius scytale (Linnaeus, 1758)

BOIDAE

122. Boa constrictor Linnaeus, 1758

123. Corallus hortulanus (Linnaeus, 1758)

124. Eunectes murinus (Linnaeus, 1758) 


\section{COLUBRIDAE}

125. Chironius carinatus (Linnaeus, 1758)

126. Chironius fuscus (Linnaeus, 1758)

127. Chironius scurrulus (Wagler, 1824)

128. Drymarchon corais (Boie, 1827)

129. Drymobius rhombifer (Günther, 1860)

130. Drymoluber dichrous (Peters, 1863)

131. Leptophis ahaetulla (Linnaeus, 1758)

132. Oxybelis fulgidus (Daudin, 1803)

133. Pseustes poecilonotus (Günther, 1858)

134. Spilotes pullatus (Linnaeus, 1758)

135. Tantilla melanocephala (Linnaeus, 1758)

Dipsadidae

136. Atractus major Boulenger, 1894

137. Atractus schach (Boie, 1827)

138. Clelia clelia (Daudin, 1803)

139. Dipsas catesbyi Schlegel, 1837

140. Dipsas indica Laurenti, 1768

141. Drepanoides anomalus (Jan, 1863)

142. Helicops angulatus (Linnaeus, 1758)

143. Imantodes cenchoa (Linnaeus, 1758)

144. Leptodeira annulata (Linnaeus, 1758)

145. Liophis dorsocorallinus Esqueda, Natera, La Marca \& Ilija-Fistar, 2005

146. Liophis reginae (Linnaeus, 1758)

147. Liophis taeniogaster Jan, 1863

148. Liophis typhlus (Linnaeus, 1758)

149. Oxyrhopus melanogenys (Tschudi, 1845)

150. Oxyrhopus occipitalis Wagler, 1824

151. Oxyrhophus petola (Linnaeus, 1758)

152. Philodryas argentea (Daudin, 1803)

153. Siphlophis compressus (Daudin, 1803)

154. Taeniophallus brevirostris (Peters, 1863)

155. Umbrivaga pygmaea (Cope, 1868)

156. Xenodon severus (Linnaeus, 1758)

157. Xenopholis scalaris (Wücherer, 1861)

Elapidae

158. Micrurus hemprichii (Jan, 1858)

159. Micrurus lemniscatus (Linnaeus, 1758)

160. Micrurus remotus Roze, 1987

Viperidae

161. Bothrops atrox (Linnaeus, 1758)

162. Lachesis muta (Linnaeus, 1766)

\section{Discussão}

Foram registradas 162 espécies de anfíbios e répteis para a área do Igarapé Esperança na Resex Riozinho da Liberdade. As curvas acumuladas de espécies indicaram que os anuros e lagartos foram bem amostrados, estando suas riquezas ( 80 espécies de anuros e 29 de lagartos) pelo tamanho da área amostrada dentro do esperado em localidades amazônicas (Duellman, 1990, Lima et al. 2006, Bernarde 2007, Macedo et al. 2008, Avila-Pires et al. 2009). Para serpentes, é provável que ocorram mais espécies na localidade de estudo devido o fato da riqueza registrada (42 espécies) ser inferior ao que é conhecido geralmente em localidades amazônicas (Duellman 1990, Jorge-da-Silva Junior 1993, Martins \& Oliveira 1998, Bernarde \& Abe 2006). Para se amostrar bem comunidades de serpentes na Amazônia, é necessário estudos com maior esforço amostral e com duração de pelo menos dois anos (e. g., Martins \& Oliveira 1998, Bernarde \& Abe 2006).
A salamandra que não foi possível a identificação a nível específico (Bolitoglossa sp.) pode ser novo registro para o Brasil ou ser uma forma ainda não descrita. A salamandra conhecida para o Brasil é B. paraensis, entretanto existe pelo menos uma espécie ainda não descrita na Amazônia brasileira (Avila-Pires et al. 2007). O dendrobatídeo Adelphobates sp.n. trata-se de uma espécie em fase de descrição por Grant, Bernarde, Machado \& Turci.

Foram registradas pela primeira vez para o Acre 11 espécies da herpetofauna (Adelphobates quinquevittatus, Hyalinobatrachium munozorum, Pristimantis academicus, P. aureolineatus, Syncope antenori, Alopoglossus buckleyi, Drymobius rhombifer, Liophis dorsocorallinus, L. taeniogaster, Umbrivaga pygmaea e Micrurus remotus), sendo que seis delas (H. munozorum, P. academicus, P. aureolineatus, S. antenori, A. buckleyi $L$. dorsocorallinus) consistem também os primeiros registros para o Brasil.

O dendrobatídeo Adelphobates quinquevittatus era conhecido para bacia do Rio Madeira nos estados de Rondônia e Amazonas (Caldwell \& Myers 1990) e na Bolívia (De La Riva et al. 2000). Esse registro no Noroeste do Brasil para a Resex Riozinho da Liberdade amplia em aproximadamente 630 e $780 \mathrm{~km}$ das localidades mais próximas conhecidas no Departamento Pando na Bolívia (De La Riva et al. 2000) e o Igarapé Puruzinho no Amazonas (Caldwell \& Myers 1990), respectivamente.

Hyalinonatrachium munozorum ocorre no Equador e Colômbia (Frost 2010), ampliando nesse trabalho em aproximadamente $1.000 \mathrm{~km}$ para o sudeste da localidade mais próxima (Santa Cecília, Equador).

Pristimantis academicus foi recentemente descrita por Lehr et al. (2010) para Puerto Almendreas (Departamento de Loreto) no Peru, localidade situada aproximadamente $260 \mathrm{~km}$ ao noroeste da Resex Riozinho da Liberdade.

Pristimantis aureolineatus é uma espécie que vive nas copas das árvores e dificilmente é coletada (Guayasamin et al. 2006), ocorrendo no Equador e Peru. O registro dessa espécie amplia em aproximadamente $530 \mathrm{~km}$ para o sudeste da localidade mais próxima conhecida (Departamento de Loreto, Peru).

Syncope antenori é um Microhylidae conhecido para Napo e Pastaza no Ecuador e para Loreto e Madre de Dios no Peru (Frost, 2010). Com esse novo registro amplia a área de ocorrência dessa espécie em aproximadamente $470 \mathrm{~km}$ ao norte de Madre de Dios e $750 \mathrm{~km}$ ao sul de Loreto (PE), que são as localidades mais próximas com ocorrência conhecida.

Três espécies de anuros (Ameerega macero, Pristimantis acuminatus e $P$. diadematus) registrados anteriormente para a Serra do Divisor no Acre (Souza 2009), representam o segundo registro para o Brasil. Phyllomedusa atelopoides ocorre no Peru e no Brasil era conhecida para a Serra do Divisor (Souza 2009) e para Coari no Amazonas (Peloso et al. 2009). Pristimantis reichlei era conhecida apenas para o sul do Acre no município de Senador Giomard (Sampaio \& Souza 2010), ampliando sua distribuição nesse estudo em aproximadamente $600 \mathrm{~km}$ para o norte do estado.

O lagarto Alopoglossus buckleyi ocorre no Equador, Colômbia e Peru, sendo conhecido na divisa entre o Acre e Peru, entretanto com nenhum espécime sido mencionado em literatura para o Brasil (Avila-Pires 1995), com esse registro na Resex Riozinho da Liberdade é confirmada sua ocorrência para o país.

Registra-se pela primeira vez para o Brasil a serpente Liophis dorsocorallinus, que era conhecida para Reserva Florestal de Caparo no sudoeste da Venezuela (Esqueda et al. 2005), região que dista aproximadamente $1700 \mathrm{~km}$ ao norte da Resex Riozinho da Liberdade. Essa espécie foi coletada também por França \& Venâncio (2010) em Boca do Acre (Amazonas) e identificada como Liophis sp. 
Quatro espécies de serpentes (Drymobius rhombifer, Liophis taeniogaster, Umbrivaga pygmaea e Micrurus remotus) foram registradas pela primeira vez para o Acre. A serpente D. rhombifer foi registrada pela quarta vez para o Brasil, sendo conhecida para o Amapá (O'Shea \& Stimson 1993) e Rondônia (Vanzolini 1986, Bernarde \& Abe 2006) e agora para o Acre. A serpente L. taeniogaster tem sua distribuição para o Peru, Colômbia, Bolívia e no Brasil ocorre do Nordeste do estado da Bahia para o Pará, Amazonas, Mato Grosso, Rondônia e Amapá (Fernandes et al. 2002). Com poucos exemplares colecionados, a serpente $U$. pygmaea é conhecida para o Peru (Dixon \& Soini 1986) e no Brasil no Amazonas na região de Manaus (Martins \& Oliveira 1998) e em Tefé (Fernandes et al. 1999), e no Pará em Oriximiná (Avila-Pires et al. 2010). M. remotus ocorre do sul da Venezuela, leste da Colômbia e no Brasil no oeste do Amazonas (Campbell \& Lamar 2004).

Além da riqueza de anfíbios e répteis registrada para uma pequena área amostrada (200 ha) da Reserva Extrativista do Riozinho da Liberdade nesse estudo, foram também realizados novos registros para o Acre e para o Brasil, além da descoberta de uma espécie não descrita. Isso denota a necessidade de estudos para a região do Alto Juruá e a importância dessa região para conservação da herpetofauna. O Alto Juruá é a região com menor índice de desmatamento no estado do Acre (Silva \& Ribeiro 2004) e isso esta associado principalmente a presença de várias unidades de conservação (parque nacional, florestas estaduais, reservas extrativista), terras indígenas e as atividades extrativistas dos povos que habitam essa região (Brown Junior \& Freitas 2002, Almeida 2004). O avanço da pecuária e da soja nos estados de Mato Grosso, Rondônia e Pará, contribui significativamente para o desmatamento na Amazônia (Ferreira et al. 2005, Rivero et al. 2009) e isso ocasiona uma ameaça à biodiversidade e aos serviços ambientais dessa região (Fearnside 2008, Vieira et al. 2008). A transformação de florestas em áreas de pastagem ocasiona uma diminuição da riqueza de espécies da herpetofauna (Bernarde 2007, Bernarde \& Macedo 2008, Macedo et al. 2008), como é observado em Rondônia onde a pecuária é uma das principais atividades econômicas. Salienta-se aqui a importância da forma do uso das florestas pelas populações tradicionais (indígenas, extrativistas e ribeirinhos), como ocorre na Reserva Extrativista do Riozinho da Liberdade, e das áreas protegidas na conservação da alta biodiversidade encontrada no Alto Juruá e também a necessidade de estudos sobre o uso de algumas espécies (especialmente de quelônios e crocodilianos) para analisar possíveis impactos sobre as populações desses animais.

\section{Agradecimentos}

Ao Daniel Loebmann pela leitura e sugestões feitas para esse manuscrito. Aos ajudantes de campo Cleuson Juvêncio Barroso (Petelti), Antonio Leidinaldo da Silva (Dinda) e José Pereira das Neves (Zé Gordo). Ao Adriano Oliveira Maciel pela identificação dos gimnofionos. Ao Diego Francisco Cisneros Heredia pela identificação de Hyalinobatrachium munozorum. Ao Maurício Forlani pela identificação de Syncope antenori. Ao Paulo Passos pela identificação de Atractus schach. Ao Mário R. Moura pela elaboração do mapa. Ao Centro de Conservação e Manejo de Répteis e Anfíbios - RAN e o SISBIO do IBAMA pelas licenças de coleta concedidas ( $\mathrm{n}^{\circ} 12178-1$ e 12178-2). Ao Francisco Barbosa de Melo (Chico Ginú) e demais funcionários do Instituto Chico Mendes e do Ibama de Cruzeiro do Sul pelo apoio. À Fundação O Boticário de Proteção à Natureza pelo patrocínio concedido (Projeto ${ }^{\circ}$ 0707-20061). Ao CNPq pelas bolsas de produtividade em pesquisa para PSB (501927/2009-3) e para RAM (502736/2009-7), e pela bolsa de Mestrado para LCBT.

\section{Referências Bibliográficas}

ACRE (Estado). 2006. Programa Estadual de Zoneamento Ecológico Econômico do Estado do Acre Fase II. Rio Branco, SEMA, Escala $1: 250.000,356 \mathrm{p}$

ALMEIDA, M.W.B. 2004. Direitos à floresta e ambientalismo: seringueiros e suas lutas. Rev. Bras. Cienc. Soc. 19 (55):33-52.

AVILA-PIRES, T.C.S. 1995. Lizards of brazilian Amazonian (Reptilia: Squamata). Zool. Verh. Leiden 299:1-706.

AVILA-PIRES, T.C.S. \& HOOGMOED, M.S. 1997. The herpetofauna. In Caxiuanã: Desafios para a Conservação de uma Floresta Nacional na Amazônia (P.L.B. Lisboa, org.). MPEG, Belém, p.389-401.

AVILA-PIRES, T.C.S., HOOGMOED, M.S. \& ROCHA, W.A. 2010. Notes on the Vertebrates of northern Pará, Brazil: a forgotten part of the Guianan Region, I. Herpetofauna. Boletim do Museu Paraense Emílio Goeldi. Sér. Ciênc. Nat. v. 5, p. 13-112.

AVILA-PIRES, T.C.S., HOOGMOED, M.S. \& VITT, L.J. 2007. Herpetofauna da Amazônia. Herpetologia no Brasil II (In L.B. Nascimento \& M.E. Oliveira, eds.). Sociedade Brasileira de Herpetologia, Belo Horizonte, p.13-43.

AVILA-PIRES, T.C.S. \& VITT, L.J. 1998. A new species of Neusticurus (Reptilia: Gymnophthalmidae) from the Rio Juruá, Acre, Brazil. Herpetologica. 54:235-245.

AVILA-PIRES, T.C.S., VITT, L.J., SARTORIUS, S.S. \& ZANI, P.A. 2009. Squamata (Reptilia) from four sites in southern Amazonia, with a biogeographic analysis of Amazonian lizards. Bol.Mus. Para. Emílio Goeldi Ciênc. Nat. 4(2):99-118.

AZEVEDO-RAMOS, C. \& GALLATI, U. 2001. Relatório técnico sobre a diversidade de anfíbios na Amazônia brasileira. Biodiversidade na Amazônia Brasileira. In Avaliação e ações prioritárias para a conservação, uso sustentável e repartição de benefícios (J.P.R. Capobianco Org.) Estação Liberdade, Instituto Socio-Ambiental, São Paulo, p.79-88.

BERGMANN, P.J. \& RUSSEL, A.P. 2007. Systematics and biogeography of the widespread Neotropical gekkonid genus Thecadactylus (Squamata), with the description of a new cryptic species. Zool. J. Linn. Soc. 149:339-370

BERNARDE, P.S. 2007. Ambientes e temporada de vocalização da anurofauna no Município de Espigão do Oeste, Rondônia, Sudoeste da Amazônia - Brasil (Amphibia: Anura). Biota Neotrop. 7(2): http://www. biotaneotropica.org.br/v7n2/pt/abstract?article+bn01507022007 (último acesso em 20/10/2010).

BERNARDE, P.S. \& ABE, A.S. 2006. A snake community at Espigão do Oeste, Rondônia, Southwestern Amazon, Brazil. South Am. J. Herpetol. 1(2):102-113. http://dx.doi.org/10.2994/18089798(2006)1[102:ASCAED]2.0.CO;2

BERNARDE, P.S. \& MACEDO, L.C. 2008. Impacto do desmatamento e formação de pastagens sobre a anurofauna de serapilheira em Rondônia. Iheringia. 98(4):454-459.

BERNARDE, P.S., MIRANDA, D.B., ALBUQUERQUE, S. \& TURCI, L.C.B. 2010. Amphibia, Anura, Hemiphractidae, Hemiphractus helioi Sheil and Mendelson, 2001: distribution extension in the state of Acre and second record for Brazil. Check List. (4):491-492.

BÉRNILS, R.S. 2010. Brazilian reptiles - List of species. Sociedade Brasileira de Herpetologia. http://www.sbherpetologia.org.br/ (último acesso em $13 / 10 / 2010)$

BROWN JUNIOR, K.S. \& FREITAS, A.V.L. 2002. Diversidade Biológica no Alto Juruá: Avaliação, Causas e Manutenção. In Enciclopédia da Floresta: O Alto Juruá: Práticas e Conhecimentos das Populações. (M.C. Cunha \& M.B. Almeida, orgs.). Companhia das Letras, São Paulo, p.33-42.

CALDWELL, J.P. 2005. A new Amazonian species of Cryptophyllobates (Anura: Dendrobatidae). Herpetologica 61(4):449-461. http://dx.doi. org/10.1655/04-86.1

CALDWELL, J.P. \& MYERS, C.W. 1990. A new poison frog from Amazonian Brazil, with further revision of the quinquevittatus group of Dendrobates. Am. Mus. Novittates. 2988:1-21. 
CAMPBELL, H.W. \& CHRISTMAN, S.P. 1982. Field techniques for herpetofaunal community analysis. In Herpetological communities: a Symposium of the Society for the Study of Amphibians and Reptiles and the Herpetologis's League (N.J. Scott Junior, ed.). U.S. Fish Wildlife Service, Washington, p.193-200.

CAMPBELL, J.A. \& LAMAR, W.W. 2004. The venomous reptiles of Latin América. Cornell Univ. Press, Ithaca, 425p.

CARDOSO, A.J.\& VIELLIARD, J. 1990. Vocalizações de anfíbios anuros de um ambiente aberto, em Cruzeiro do Sul, Estado do Acre. Rev. Bras. Biol. 50:229-242.

CASTROVIEJO-FISHER, S., PADIAL, J.M., CHAPARRO, J.C., AGUAYO, R. \& DE LA RIVA, I. 2009. A new species of Hyalinobatrachium (Anura: Centrolenidae) from the Amazonian slopes of the central Andes, with comments on the diversity of the genus in the area. Zootaxa. 2143:24-44.

CECHIN, S.Z. \& MARTINS, M. 2000. Eficiência de armadilhas de queda (pitfall traps) em amostragem de anfíbios e répteis no Brasil. Rev. Bras. Zool. 17:729-740. http://dx.doi.org/10.1590/S0101-81752000000300017

CISNEROS-HEREDIA, D.F. \& McDIARMID, R.W. 2007. Revision of the characters of Centrolenidae (Amphibia: Anura: Athesphatanura), with comments on its taxonomy and the description of new taxa of glassfrogs. Zootaxa. 1572:1-82.

CISNEROS-HEREDIA, D.F., STRUSSMANN, C., ÁVILA, R.W. \& KAWASHITA-RIBEIRO, R.A. 2010. Amphibia, Anura, Centrolenidae, Hyalinobatrachium carlesvilai Castroviejo-Fisher, Padial, Chaparro, Aguayo \& De La Riva, 2009: First country record, Brazil. Check List. 6(2):225-226

CUNHA, O.R. \& NASCIMENTO, F.P. 1993. Ofídios da Amazônia: as cobras da região Leste do Pará. Bol. Mus. Para. Emílio Goeldi., Sér. Zool. 9(1):1-191.

DE LA RIVA, I., KOHLER, J., LOTTERS, S. \& REICHLE, S. 2000. Ten years of research on Bolivian amphibians: Update checklist, distribution, taxonomic problems, literature and iconography. Rev. Esp. Herpetol. 14:19-164.

DIXON, J.R. \& SOINI, P. 1986.The reptiles of the upper Amazon basin, Iquitos region, Peru. 2nd ed. Milwaukee Public Museum, Milwaukee.

DUELLMAN, W.E. 1978. The biology of na equatorial herpetofauna in Amazonian Equador. Misc. Pub. Mus. Nat. Hist. Univ. Kansas. 65:1-352.

DUELLMAN, W.E. 1990. Herpetofaunas in Neotropical rainforests: comparative composition, history, and resource use. In Four Neotropical Rainforests (A. H. Gentry, ed.). Yale University Press, New Haven, p.455-505.

DUELLMAN, W.E. \& RODRIGUEZ, L.O. 1994. Guide to the Frogs of the Iquitos Region, Amazonian Peru. Lawrence, Kansas. Asociación de Ecologia y Conservación, Amazon Center for Environmental Education and Research, and Natural History Museum, The University of Kansas, p. 80.

ESQUEDA, L.F., NATERA, M., LA MARCA, E. \& ILIJA-FISTAR, M. 2005. Nueva especie de serpente (Reptilia: Colubridae: Liophis) de un bosque tropical relictual en el estado Barinas, Venezuela. Herpetotropicos 2(2):95-103.

FACHÍN-TERÁN, A., VOGT, R.C. \& THORBJARNARSON, J.B. 2003. Estrutura populacional, razão e abundância de Podocnemis sextuberculata (Testudines, Podocnemididae) na Reserva de Desenvolvimento Sustentável de Mamirauá, Amazonas, Brasil. Phyllomedusa. 2(1):43-63.

FEARNSIDE, P.M. 2006. Desmatamento na Amazônia: dinâmica, impactos e controle. Acta Amazon. 36(3):395-400. http://dx.doi.org/10.1590/ S0044-59672006000300018

FEARNSIDE, P.M. 2008. Amazon forest maintenance as a source of environmental services. An. Acad. Bras. Cienc. 80(1):101-114. http:// dx.doi.org/10.1590/S0001-37652008000100006

FERNANDES, D.S., FRANCO, F.L. \& GERMANO, V.J. 1999. Geographic distribution. Umbrivaga pygmaea. Herpetol. Rev. 30(3):175.

FERNANDES, D.S., GERMANO, V.J., FERNANDES, R. \& FRANCO, F.L. 2002. Taxonomic status and geographic distribution of the lowland species of the Liophis cobella group with comments on the species from the Venezuelan tepuis (Serpentes, Colubridae). Bol. Mus. Nac., Zool. 481:1-14.

FERREIRA, L.V., VENTICINQUE, E. \& ALMEIDA, S. 2005. O desmatamento na Amazônia e a importância das áreas protegidas. Estud. Av. 19(53):157-166.
FITCH, H.S. 1987. Collecting and life-history techniques: Snakes, Ecology and evolutionary biology (In Seigel, R.A., Collins, J.T. \& Novak, S.S, eds.). MacMillan Publishing Company, New York, p.143-164.

FRANÇA, F.G.R. \& VENÂNCIO, N.M. 2010. Reptiles and amphibians of a poorly known region in southeastern Amazonia. Biotemas. 23(3):71-84.

FROST, D.R. 2010. Amphibian species of the world: an online reference. Version 5.4. American Museum of Natural History, New York. http:// research.amnh.org/vz/herpetology/amphibia/ (último acesso em 13/10/2010).

FUnK, W.C., ANGUlO, A., CALDWELL, J.P., RYAN, M.J. \& CANNATELLA, D.C. 2008. Comparison of morphology and calls of two cryptic species of Physalaemus (Anura: Leiuperidae). Herpetologica. 64(3):290-304. http://dx.doi.org/10.1655/08-019.1

GUAYASAMIN, J.M., RON, S.R., CISNEROS-HEREDIA, D.F., LAMAR, W. \& McCRACKEN, S.F. 2006. A new species of frog of the Eleutherodactylus lacrimosus assemblage (Leptodactylidae) from the western Amazon basin, with comments on the utility of canopy surveys in lowland rainforest. Herpetologica. 62(2):191-202. http://dx.doi. org/10.1655/05-40.1

HEYER, R.H., DONNELLY, M.A., MCDIARMID, R.W., HAYEK, L.C. \& FOSTER, M.S. 1994. Measuring and monitoring biological diversity: Standard methods for amphibians. Smithsonian Institution Press, Washington, 364p.

JORGE-DA-SILVA JUNIOR, N. 1993. The snakes from Samuel hydroeletric power plant and vicinity, Rondônia, Brasil. Herpetol. Nat. Hist. 1(1):37-86.

LEHR, E., MORAVEC, J. \& URRUTIA, L.A.G. 2010. A new species of Pristimantis (Anura: Strabomantidae) from the Amazonian lowlands of northern Peru. Salamandra 46(4):197-203.

LIMA, A.C. \& PRUDENTE, A.L.C. 2009. Morphological variation and systematics of Dipsas catesbyi (Sentzen, 1796) and Dipsas pavonina Schlegel, 1837 (Serpentes: Dipsadinae). Zootaxa. 2203:31-48.

LIMA, A.P., MAGNUSSON, W.E., MENIN, M., ERDTMANN, L.K., RODRIGUES, D.J., KELLER, C. \& HÖDL, W. 2006. Guia de sapos da Reserva Adolph Ducke - Amazônia Central. Editora Attema, INPA, Manaus, 168p.

LYNCH, J.D. 2009. Snakes of the genus Oxyrhopus (Colubridae: Squamata) in Colombia: taxonomy and geographic variation. Pap. Avul. Zool. 49(25):319-337.

MACEDO, L.C., BERNARDE, P.S. \& ABE, A.S. 2008. Lagartos (Squamata:Lacertilia) em áreas de floresta e de pastagem em Espigão do Oeste, Rondônia, sudoeste da Amazônia, Brasil. Biota Neotrop. 8(1): http://www.biotaneotropica.org.br/v8n1/pt/fullpaper?bn01108012008 (último acesso em 13/10/2010).

MARTINS, M. 1991. The lizards of balbina, Central Amazonia, Brazil. Stud. Neotrop. Fauna Environ. 26:179-190. http://dx.doi. org/10.1080/01650529109360851

MARTINS, M. \& CARDOSO, A.J. 1987. Novas espécies de hilídeos do Estado do Acre (Amphibia: Anura). Rev. Bras. Biol. 47: 549-558.

MARTINS, M. \& OLIVEIRA, M.E. 1998. Natural history of snakes in forests of the Manaus region, Central Amazonia, Brazil. Herpetol. Nat. Hist. 6(2):78-150.

MASCHIO, G.F., SANTOS-COSTA., M.C., PRUDENTE, A.L.C. 2009. Comunidades de Serpentes da região de Caxiuanã com avaliação da eficiência dos métodos de captura. In Caxiuanã: Desafios para a Conservação de uma Floresta Nacional na Amazônia (P.L.B. Lisboa, org.). MPEG, Belém, p.589-603.

MENIN, M., LIMA, A.P., MAGNUSSON, W.E. \& WALDEZ, F. 2007. Topographic and edaphic effects on the distribution of terrestrially reproducing anurans in Central Amazonia: mesoscale spatial patterns. J. Trop. Ecol. 23:539-547. http://dx.doi.org/10.1017/S0266467407004269

NASCIMENTO, F.P., ÁVILA-PIRES, T.C.S.\& CUNHA, O.R. 1988. Répteis Squamata de Rondônia e Mato Grosso coletados através do programa Polonoroeste. Bol. Mus. Para. Emílio Goeldi, ser. Zool. 4:21-66. 
O'SHEA, M.T. \& STIMSON, A.F. 1993. An aberrant specimen of Drymobius rhombifer (Colubridae: Colubrinae): a new generic record for Brazil. Herpetol. J. 3:70-71.

PADIAL, J.M. \& DE LA RIVA, I. 2009. Integrative taxonomy reveals cryptic Amazonian species of Pristimantis (Anura: Strabomantidae). Zool. J. Linn. Soc. 155:97-122. http://dx.doi.org/10.1111/j.10963642.2008.00424.x

PASSOS, P. \& FERNANDES, D.S. 2005. Variation and taxonomic status of the aquatic coral snake Micrurus surinamensis (Cuvier, 1817) (Serpentes: Elapidae). Zootaxa. 953:1-14.

PELOSO, P.L.V.\& STURARO, M.J. 2008. A new species of narrow-mounthed frog of the genus Chiasmocleis Méhelÿ 1904 (Anura, Microhylidae) from the Amazonian rainforest of Brazil. Zootaxa. 1947:39-52.

PELOSO, P.L., STURARO, M.J., MASCHIO, G.F., RODRIGUES, F.S. \& PRUDENTE, A.L.C. 2009. New records and distribution of the toady leaf frog Phyllomedusa atelopoides (Anura, Hylidae). Herpetol. Notes. 2:59-62.

PERES, C.A. 2000. Effects of subsistence hunting on vertebrate community structure in Amazonian forests. Conserv. Biol. 14(1):240-253. http:// dx.doi.org/10.1046/j.1523-1739.2000.98485.x

PRUDENTE, A.L.C. \& SANTOS COSTA, M.C. 2005. Checklist of Snakes in the Eastern Amazon, Pará State, Brazil: Floresta Nacional de Caxiuanã Bol. Mus. Para. Emilio Goeldi. Zoologia. 3(1):243-251.

PRUDENTE, A.L.C., SILVA, M.A.A., ROCHA, W.A. \& FRANCO, F.L. 2008. Morphological variation in Xenoxybelis boulengeri (Procter, 1923) (Serpentes, Xenodontinae, Philodryadini). Zootaxa. 1743:53-61.

RIBEIRO, A.G. 1977. O Clima do Estado do Acre. Bol. Geogr. 35:112-141.

RIVERO, S., ALMEIDA, O., ÁVILA, S. \& OLIVEIRA, W. 2009. Pecuária e desmatamento: uma análise das principais causas diretas do desmatamento na Amazônia. Nova Econ. 19(1):41-66. http://dx.doi.org/10.1590/S010363512009000100003

RYLANDS, A.B. \& BRANDON, K. 2005. Unidades de conservação brasileiras. Megadiversidade. 1(1):27-35.

SAMPAIO, P.R.M. \& SOUZA, M.B. 2010. Amphibia, Anura, Strabomantidae, Pristimantis reichlei Padial \& De La Riva, 2009: first record from Brazil, southwestern Amazonia. Check List. 6(3):385-386.

SILVA, R.G. \& RIBEIRO, C.G. 2004. Análise da degradação ambiental na Amazônia ocidental: um estudo de caso dos municípios do Acre. Rev. Econ. Sociol. Rural 42(1):91-110.

SIMÕES, P.I., LIMA, A.P. \& FARIAS, I.P. 2010. The description of a cryptic species related to the pan-Amazonian frog Allobates femoralis (Boulenger 1883) (Anura: Aromobatidae). Zootaxa. 2406:1-18.

SOCIEDADE BRASILEIRA DE HERPETOLOGIA - SBH. 2010. Brazilian amphibians - List of species. Sociedade Brasileira de Herpetologia. http:// www.sbherpetologia.org.br (último acesso em 13/10/2010).
SOUZA, M.B. 2009. Anfíbios: Reserva Extrativista do Alto Juruá e Parque Nacional da Serra do Divisor, Acre. IFCH, Campinas, 77p.

SOUZA, M.B. \& HADDAD, C.F.B. 2003. Redescription and revaluation of the generic status of Leptodactylus dantasi (Amphibia, Anura, Leptodactylidae), and description of its unusual advertisement call. J. Herpetol. 37(3):490-497. http://dx.doi.org/10.1670/259-01A

TOLEDO, L.F., ARAÚJO, O.G.S., ÁVILA, R. W., KAWASHITA-RIBEIRO, R.A., MORAIS, D.H. \& CISNEROS-HEREDIA, D.F. 2009. Amphibia, Anura, Centrolenidae, Cochranella adenocheira: distribution and range extension, Brazil. Check List. 5(3):380-382.

TURCI, L.C.B., ALBUQUERQUE, S., BERNARDE, P.S. \& MIRANDA, D.B. 2009. Uso do hábitat, atividade e comportamento de Bothriopsis bilineatus e de Bothrops atrox (Serpentes: Viperidae) na floresta do Rio Moa, Acre, Brasil. Biota Neotrop. 9(3):197-206. http://www.biotaneotropica.org.br/ v9n3/pt/abstract?article+bn03909032009 (último acesso em 13/10/2010).

VANZOLINI, P.E. 1986. Levantamento herpetológico da área do Estado de Rondônia sob a influência da rodovia Br-364. Ministério da Ciência e Tecnologia, Brasília, 50 p. (Relatório de pesquisa, Programa Polonoroeste/Ecologia Animal n. 1). VANZOLINI, P.E. 2002. An aid to the identification of the South American species of Amphisbaena (Squamata, Amphisbaenidae). Pap. Avul. Zool. 42(15):351-362.

VIEIRA, I.C.G., TOLEDO, P.M., SILVA, J.M.C. \& HIGUSHI, H. 2008. Deforestation and threats to the biodiversity of Amazonia. Braz. J. Biol. 68(4):949-956. http://dx.doi.org/10.1590/S1519-69842008000500004

VITT, L.J., AVILA-PIRES, T.C.S., CALDWELL, J.P. \& OLIVEIRA, V.R.L. 1998. The impact of individual tree harvesting on thermal environments of lizards in amazonian rain forest. Conserv. Biol. 12:654-664. http:// dx.doi.org/10.1046/j.1523-1739.1998.96407.x

VITT, L.J., MAGNUSSON, W.E., AVILA-PIRES, T.C. \& LIMA, A.P. 2008. Guia de lagartos da Reserva Adolpho Ducke: Amazônia Central. Editora Attema, INPA, Manaus, 176p.

VOGT, R.C., MOREIRA, G. \& DUARTE, A.C.O.C. 2001. Biodiversidade de répteis do bioma floresta Amazônica e Ações prioritárias para sua conservação. In: Biodiversidade na Amazônia Brasileira, Avaliação e ações prioritárias para a conservação, uso sustentável e repartição de benefícios (J.P.R. Capobianco, org.). Estação Liberdade: Instituto Socioambiental, São Paulo, p.89-96.

WHITEMAN, C.W., PALHA, M.D.C., MATUSHIMA, E.R., SILVA, A.S.L. \& MONTEIRO, V.C. 2008. Interface entre carnívoros domésticos e silvestres em área de proteção ambiental na Amazônia brasileira: indicadores e implicações para conservação. Nat. Conserv. 6(1):56-64.

ZIMMERMAN, B.L. \& RODRIGUES, M.T. 1990. Frogs, snakes, and lizards of the INPA/WWF reserves near Manaus, Brazil. In Four Neotropical Rainforests (A.H. Gentry, ed.). Yale University Press, New Haven, p.426-454. 


\section{Apêndice}

AMPHIBIA - ANURA: AROMOBATIDAE: Allobates femoralis (UFACF: 394, 524, 819, 870, 877, 879, 893, 900, 1761); Allobates sp.1 (UFACF: 138, 172, 390, 391, 558, 805, 814, 815, 821, 905, 1767); Allobates sp.2 (UFACF: 601, 638, 773); BUFONIDAE: Dendrophryniscus minutus (UFACF: 630, 640, 1756, 1782); Rhaebo guttatus (UFACF: 867); Rhinella castaneotica (UFACF: 872); Rhinella margaritifera (UFACF: 207); Rhinella sp. (UFACF: 160, 621, 627, 679, 878, 903, 925); CENTROLENIDAE: Hyalinobatrachium munozorum (UFACF: 854); CERATOPHRYIDAE: Ceratophrys cornuta (UFACF: 892); DENDROBATIDAE: Adelphobates quinquevittatus (UFACF: 822); Adelphobates sp.n (UFACF: 714, 721, 729, 732, 917); Ameerega hahneli (UFACF: 625, 631, 813, 856, 857, 864, 1763); Ameerega macero (UFACF: 698, 706, 901, 1751); Ameerega trivittata (UFACF: 781, 808, 809, 904); Ranitomeya ventrimaculata (UFACF: 546, 712, 848, 907); HEMIPHRACTIDAE: Hemiphractus scutatus (UFACF: 702, 873); HYLIDAE: Dendropsophus acreanus (UFACF: 690); Dendropsophus brevifrons (UFACF: 580, 581, 582, 583, 824, 829, 1337, 1338); Dendropsophus leali (UFACF: 826); Dendropsophus leucophyllatus (UFACF: 920); Dendropsophus nanus (UFACF: 839); Dendropsophus parviceps (UFACF: 411, 632, 849); Dendropsophus rhodopeplus (UFACF: 755, 793, 795, 830, 1786, 1791); Dendropsophus sarayacuensis (UFACF: 694); Dendropsophus triangulum(UFACF: 827, 831, 834, 835, 838, 840, 843, 846, 966); Hypsiboas boans (UFACF: 1344); Hypsiboas cinerascens (UFACF: 579, 1757, 1762); Hypsiboas fasciatus (UFACF: 401, 841, 855, 869, 1341); Hypsiboas geographicus (UFACF: 836, 1759, 1766); Hypsiboas lanciformis (UFACF: 593, 812); Osteocephalus buckleyi (UFACF: 587, 1347); Osteocephalus leprieuri (UFACF: 588); Osteocephalus taurinus (UFACF: 1339, 1753); Phyllomedusa atelopoides (UFACF: 860, 874, 1336); Phyllomedusa tarsius (UFACF: 765); Phyllomedusa tomopterna (UFACF: 145); Phyllomedusa vaillantii (UFACF: 1801); Scarthyla goinorum (UFACF: 604, 825); Scinax boesemani (UFACF: 768, 772, 776, 785); Scinax cruentommus (UFACF: 916); Scinax funereus (UFACF: 578); Scinax garbei (UFACF: 659, 837, 875); Scinax ruber (UFACF: 628, 828, 833); Sphaenorhynchus dorisae (UFACF 1349); Sphaenorhynchus lacteus (UFACF: 639, 859); Trachycephalus coriaceus (UFACF: 595, 596, 603, 658, 806); Trachycephalus resinifictrix (UFACF: 687); LEIUPERIDAE: Edalorhina perezi (UFACF: 594, 778, 868, 894); Engystomops freibergi (UFACF: 590, 591, 883, 886); LEPTODACTYLIDAE: Leptodactylus andreae (UFACF: 544, 550, 567, 569, 584, 737, 820, 865, 913, 1346, 1770, 1774); Leptodactylus lineatus (UFACF: 399, 589, 742, 754, 832, 882); Leptodactylus mystaceus (UFACF: 626, 633, 762, 1758); Leptodactylus pentadactylus (UFACF: 926); Leptodactylus petersii (UFACF: 415, 610, 661, 662, 909, 1340, 1754); Leptodactylus rhodomystax (UFACF: 556, 560, 561); Leptodactylus rhodonotus (UFACF: 1335); Leptodactylus wagneri (UFACF: 709); MICROHYLIDAE: Chiasmocleis bassleri (UFACF: 623, 676, 1768, 1772); Chiasmocleis ventrimaculata (UFACF: 549, 564, 611, 612, 613, 634, 641, 718, 723, 767, 810, 866, 897, 910, 927, 1342, 1758); Ctenophryne geayi (UFACF: 410, 624, 779, 885); Hamptophryne boliviana (UFACF: 398, 409, 889, 890, 891, 1765, 1779); Syncope antenori (UFACF: 660, 816, 850); STRABOMANTIDAE: Oreobates quixensis (UFACF: 396, 586, 780, 818, 898, 899, 1760); Pristimantis academicus (UFACF:
786); Pristimantis acuminatus (UFACF: 418, 545, 699, 735, 859); Pristimantis altamazonicus (UFACF: 716); Pristimantis aureolineatus (UFACF: 413); Pristimantis conspicillatus (UFACF: 673, 682, 711, 749, 802, 914); Pristimantis diadematus (UFACF: 395, 400, 568, 707); Pristimantis fenestratus (UFACF: 174, 402, 685, 689, 807, 844, 847, 852, 915); Pristimantis ockendeni (UFACF: 403, 414, 585, 674, 748, 750, 842, 845, 871, 902, 911, 1776, 1778); Pristimantis peruvianus (UFACF: 393, 881); Pristimantis reichlei (UFACF: 412, 419, 753, 863, 1348, 1752); URODELA - PLETHODONTIDAE: Bolitoglossa sp. (UFACF: 636, 728, 746, 789, 876); GYMNOPHIONA CAECILIIDAE: Caecilia tentaculata (UFACF: 679, 680, 688, 692); Caecilia marcusi (UFACF: 701). REPTILIA: SQUAMATA - AMPHISBAENIDAE: Amphisbaena fuliginosa (UFACF: 597); LACERTILIA - IGUANIDAE: Iguana iguana (UFACF: 671); HOPLOCERCIDAE: Enyalioides laticeps (UFACF: 811); Enyalioides palpebralis (UFACF: 407); POLYCHROTIDAE: Anolis fuscoauratus (UFACF: 404, 541, 675, 763, 858, 1777, 1781, 1802); Anolis nitens (UFACF: 760, 1755); Anolis ortonii (UFACF: 473, 509); Anolis trachyderma (UFACF 595, 725, 858) TROPIDURIDAE: Plica plica (UFACF: 408); Plica umbra (UFACF: 787, 1773); Stenocercus fimbriatus (UFACF: 392, 406, 791); SPHAERODACTYLIDAE: Gonatodes hasemani (UFACF: 525, 761); Gonatodes humeralis (UFACF: 600, 602, 715, 794, 803, 884); Pseudogonatodes guianensis (UFACF: 592, 614, 615, 790, 957); TEIIDAE: Ameiva ameiva (UFACF: 943); Kentropyx pelviceps (UFACF: 1345); Kentroyix altamazonica (UFACF: 853); Tupinambis teguixin (UFACF: 705); GYMNOPHTHALMIDAE: Alopoglossus buckleyi (UFACF: 572, 887); Bachia peruana (UFACF: 551, 573); Cercosaura argulus (UFACF: 948); Cercosaura ocellata (UFACF: 540, 683, 792, 797, 912); Iphisa elegans (UFACF: 503); Ptychoglossus brevifrontalis (UFACF: 397, 722, 745, 751, 895, 908). SERPENTES - ANIILIDAE: Anilius scytale (UFACF: 777); BOIDAE: Corallus hortulanus (UFACF: 738, 747, 766); COLUBRIDAE: Chironius carinatus (UFACF: 652, 684, 759, 769, 896, 928); Drymobius rhombifer (UFACF: 417, 666, 906); Drymoluber dichrous (UFACF: 663, 720, 741, 801, 880, 1333); Leptophis ahaetulla (UFACF: 756); Oxybelis fulgidus (UFACF: 783); Spilotes pullatus (UFACF: 607); Tantilla melanocephala (UFACF: 547); DIPSADIDAE: Atractus major (UFACF: 553, 616, 618, 653, 677, 686, 700, 703, 736, 757); Atractus schach (UFACF: 416); Clelia clelia (UFACF: 667); Dipsas catesbyi (UFACF: 650, 651, 656, 657, 710, 752, 796, 799, 956, 1207); Dipsas indica (UFACF: 606, 719, 960); Drepanoides anomalus (UFACF: 654, 704, 764); Helicops angulatus (UFACF: 189); Imantodes cenchoa (UFACF: 609, 670, 691, 708, 724, 730, 731, 744); Liophis dorsocorallinus (UFACF: 405, 635, 655, 664, 918); Liophis reginae (UFACF: 548); Liophis taeniogaster (UFACF: 1803); Oxyrhopus melanogenys (UFACF: 619, 693, 696, 713, 733, 774, 775, 946); Oxyrhopus petola (UFACF: 555, 570, 617, 695); Oxyrhopus occipitalis (UFACF: 770, 862, 1798); Philodryas argentea (UFACF: 665, 717, 734, 740, 743, 771, 784, 1771); Siphlophis compressus (UFACF: 739); Taeniophallus brevirostris (UFACF: 788); Umbrivaga pygmaea (UFACF: 804); Xenopholis scalaris (UFACF: 637); ELAPIDAE: Micrurus hemprichii (UFACF: 575, 727); Micrurus lemniscatus (UFACF: 668, 726); Micrurus remotus (UFACF: 605, 620, 758, 782); VIPERIDAE: Bothrops atrox (UFACF: 608, 672, 678, 1790). 